\title{
Workforce planning in a lotsizing mail processing problem
}

\author{
Joaquim Júdice ${ }^{\mathrm{a}, *}$, Pedro Martins ${ }^{\mathrm{b}}$, Jacinto Nunes ${ }^{\mathrm{c}}$ \\ ${ }^{a}$ Departamento de Matemática, FCT-Universidade de Coimbra, 3000 Coimbra, Portugal \\ ${ }^{\mathrm{b}} \mathrm{CIO}$ - Centro de Investigação Operacional_FC/UL and ISCAC_Instituto Politécnico de Coimbra, \\ Quinta Agrícola-Bencanta, 3040-316 Coimbra, Portugal \\ ${ }^{\mathrm{c}}$ CTT-Correios de Portugal, SA, Praça D. Luís I, 30, 1208-148 Lisboa, Portugal
}

Available online 9 June 2004

\begin{abstract}
The treatment of mail objects in a mail processing centre involves many operations, in particular sorting by destination. Out of the batching problem that we can identify in such a process, there are also staff planning concerns. In this paper, we analyse a treatment area (registered mail) belonging to a mail processing center, where mail objects are treated in a chain production process. The production quantities and the transfer amounts among machines are required to be determined along the daily work period. The objective is to minimize the costs with human resources needed in the process, linked with the lotsizing production plan, by matching staff to work requirements. This leads into a lotsizing and workforce problem, for which we propose an integer programming formulation. A case study of a particular treatment area is also discussed. The formulation is adjusted to the specific constraints of this case study and some computational results are included, considering average, small and high daily amounts of mail arrived to that particular treatment area. (C) 2004 Elsevier Ltd. All rights reserved.
\end{abstract}

Keywords: Workforce scheduling; Batching decision process; Mixed integer programming; Postal service

\section{Introduction}

This work addresses a particular case of a batching decision process in a mail processing center (MPC) in the Portuguese Mail Service "CTT_Correios de Portugal, SA". One of the crucial concerns of the Staff Management Department is the employees' assignment plan that is needed to cover all the mail flow in the mail treatment system, where mail-objects are sorted according to their type, final destination and duty evaluation.

\footnotetext{
* Corresponding author.

E-mail addresses: joaquim.judice@co.it.pt (J. Júdice), pmartins@iscac.pt (P. Martins), jacinto.m.nunes@ctt.pt (J. Nunes).
} 
The treatment process is made in MPCs placed in the Portuguese territory. Each MPC is divided into treatment areas (TAs), according to a specific classification of mail-objects: large mail-objects, medium mail-objects, flats, expressed mail and registered mail. In each TA, the employees' assignment plan is established for a week. In this work, we analyse the year 2001 behaviour of a particular $\mathrm{TA}$, in order to evaluate human resources needs from lower to higher daily mail amounts entered in that specific TA. Those observations are important to plan future manpower needs in that TA.

The treatment process made in each TA involves a predefined sequence of treatment units spanning all mail flow. The mail is treated within fixed periods of $15 \mathrm{~min}$, and the sequence of consecutive periods establishes a partition of the whole planning horizon. Among consecutive periods, the mail treated is transferred to a subsequent treatment unit through a well-defined sequence and according to known mail transfer rates. The determination of the amounts treated in each unit and in each period characterizes a batch decision process. All the treatment units require human resources, which must be linked with the feasible working shifts in use in the Company.

Therefore, we may identify in the process a single-item multi-level (machine) lotsizing subproblem, characterizing the batching decision process on an acyclic graph defined by the fixed sequence of treatment units in a single TA, and a shift scheduling subproblem, characterizing the staff planning concerns. It is precisely this last aspect that concentrates the objective of this paper, where workforce overall costs are required to be minimized.

Like in many applications of operations research, this case also combines known and studied central problems. The first of these subproblems is a multi-stage batch decision problem without direct setup, holding or production costs, but using the same flow conservation proprieties appearing in multi-level lotsizing problems. Many researchers have devoted attention to the central version of this optimization problem (with setup, holding and production costs) (see, e.g. [1-6]). Most of these works are motivated by production planning environments, where the multi-level structure is defined by a sequence of machines establishing a gozinto structure. There are different types of gozinto structures (see [6]), most of them describing assembly systems. In our problem we do not assemble items, but we may find in this problem an acyclic gozinto-network of the general type (see [6]), defined by the fixed sequence of treatment units (operations), through which mail-objects (a single item) pass through. A general description of various other lotsizing or batch decision related problems can be found in [7-9]. Formulations for some practical lotsizing based problems are discussed in [10]. Interesting catalogues of selected research are presented in [7,9,11] and various models and optimization methods are discussed in [10,12-15].

The second subproblem is a shift scheduling problem, also known by staff planning or workforce scheduling. It emerges from set covering optimization problems (see, [16]), and comes frequently assigned to other problems in matching staffing to work requirements, characterizing real applications. Various formulations for shift scheduling problems are compared in [17,18]. Optimization methods are discussed in $[18,19]$. A catalogue of selected research is presented in [20] and a review of applications, methods and models has been recently published in [21]. Applications are commonly described in crew scheduling for bus, rail, air flight and general transportations planning (see, e.g. [22-27]), ground station personnel scheduling at airports or maintenance teams in daily shift schedules (see, e.g. [28-30]), telecommunication services (telephone operator, telemarketing, customer hotline service) (see, e.g. [31,32]), public safety (fire, policy, emergency) (see, e.g. [33]), health care (medical, nursery shift scheduling) (see, e.g. [34,35]), and many other cases where shift scheduling planning has been revealing an important component of ongoing productivity improvement. 
Staff scheduling has also been applied to the postal sector in [36-38]. The first work [36] addresses a personnel scheduling problem for postal distribution service in the United States postal distribution stations. The authors propose an integer model for this problem, determining daily assignments of full- and part-time employees to different task categories, considering regular and overtime hours. The paper includes some computational results using real data from a typical week in a large postal distribution station.

The second work [37] addresses a daily personnel scheduling problem to establish weekly tours, involving break time periods and different shift start time periods. The problem considers full- and part-time employees and satisfies some labour requirements. The authors analyse three problems on the system; the first is concerned with the assignment of days off to employees (the days-off scheduling problem); the second determines an assignment of employees to shifts during the day (the shift scheduling problem); and the third involves the construction of weekly tours (the tour scheduling problem). The problem is motivated by general mail facilities (GMFs), which provide for mail treatment in the US Postal Service and analyses a real application in the Providence, Rhode Island GMF.

The third work [38] comes in the trend of the second one. The authors expand on the previous work model and solution techniques and incorporate several new features in their formulation. They consider a linear integer model to solve a weekly tour scheduling problem, determining minimum cost general staffing needs using both full- and part-time employees, respecting labour agreements and governmental regulations. Different scenarios are examined, including different days off, variable start times and the use of part-time flexible workers. This work has also been motivated by the United States Postal Service and applied to the Oklahoma City processing and distribution centre.

Our problem also involves the determination of an assignment of employees to shifts within a daily working time horizon, where different shifts are considered, involving different durations and start time periods. In this case, the model is not solved over known workload requirements but it must cover workloads generated by a batch decision problem running over the same time horizon. Our objective is to determine minimum cost daily workforce needs to treat mail, according to the daily amounts of mail entered in a single TA.

Many papers that consider shift scheduling as a subproblem have work requirements to be accomplished, where adequate staff coverage is required to be planned (see, e.g., the most of the papers on staff scheduling previously mentioned). One of the critics usually pointed to classical lotsizing approaches, is that it ignores, in most of the cases, that the batch decision structure is only a part of a larger global management process, turning harder to infer and analyse alternative strategies (see, e.g. [7]). The present combined approach is sensitive to this aspect, relating the two most relevant process components, namely the treatment lotsizing scheme of TAs and its staff planning concerns.

When looking from the batch decision subproblem perspective, an interesting aspect of the joint system establishes that the decision to treat mail in a certain period on a specific unit does not depend on any direct setup cost, like in lotsizing problems, but in the assigned workforce cost, defining a kind of an indirect setup cost. This means that the employees' constraints impose a workforce upper limit on mail flow in each unit and in each period, characterizing a capacitated version of the multi-level lotsizing subproblem.

In the next section, the company main features and the problem under consideration are described. A mixed integer formulation for a general treatment area on the MPC scheme is proposed in 
Section 3. A specific model for a particular treatment area case is described in Section 4, and different scenarios solutions to this case study are discussed in Section 5. Some conclusions are presented in the last section.

\section{Company overview and mail processing}

\section{1. $C T T$}

The company CTT-Correios de Portugal, SA, presents as core business the management and support of postal services over the Portuguese territory, as well as international postal services beginning or ending in Portugal.

CTT is divided into the following main job areas: home and company delivery, logistics and distribution, hybrid mail, financial services and outsourcing services offered at the post-offices all over the country. To accomplish its purposes, the company has approximately 17600 employees and owns nine mail processing centres, 418 mail distribution centres, 1079 post-offices, 19000 pillar-boxes and over 4000 vans.

Through the year 2001 over 1370 million postal-objects were processed and nearly 6300 daily postman routes were made. Money transactions exceeded 17200 million $€$, which lead the company to have more than 630 million $€$ in revenues with more than 56 million $€$ invested.

As a government-owned company, CTT is committed to accomplish quality standard criteria such as mean time to delivery. The service quality is verified by an independent sector regulator"ANACOM-Autoridade Nacional de Comunicações", which pressures CTT to accomplish with the delivery.

To plan the mail distribution process, the company divides the Portuguese territory in approximately 418 areas, each having in average $220 \mathrm{~km}^{2}$. Each of these areas has its own mail distribution centre (MDC), which receives all the mail coming from post-offices and pillar-boxes inside that area and sends it to processing centres (MPCs). The MDC also receives mail already treated and having as final destination an address inside that area. Hence, all the mail received in an MDC is sent to be treated in an MPC. MDCs also receive treated mail coming from an MPC, for distribution purposes. So, postal-objects coming from the MDCs of an area are treated (sorted) at the corresponding MPC and are dispatched from there to its destination MDC.

Postal routes, i.e., the daily journey to deliver mail from door to door, begin at the headquarters of the MDC.

The postal process consists of four main steps, namely collection, sorting by destination, transportation and delivery.

Taking into consideration the expected flow of mail to be treated and the company commitment with the quality criteria, it becomes obvious how important it is for this company to plan carefully the assignment of human resources. Although the MDCs concentrate higher manpower needs, this work focus just in the workforce planning in MPCs, where a correct assignment of employees to workload is also of a great concern, in order to accomplish with the agreed percentage of residual mail at the end of the day, according to the estimated quantity of mail received and to the targets established for quality service. 


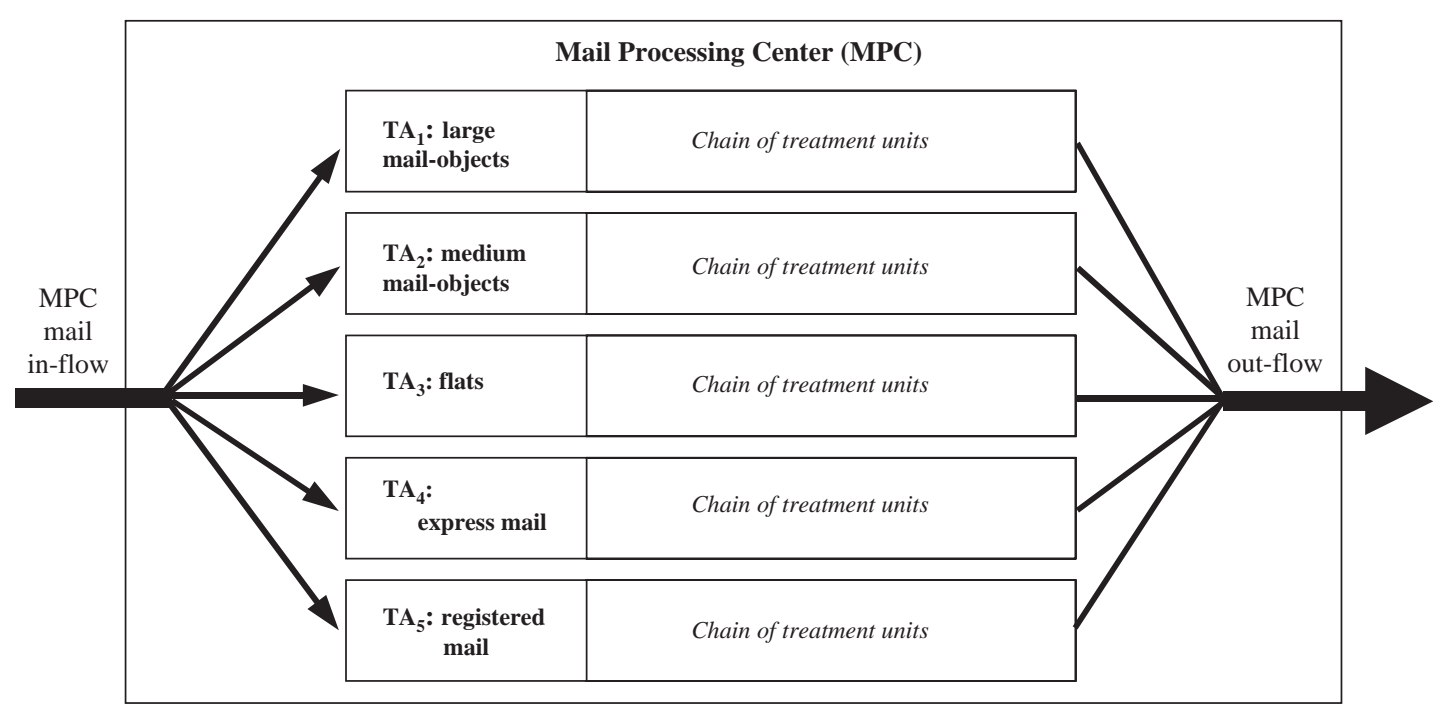

Fig. 1. Scheme of an MPC.

\subsection{The $M P C s$}

The main job of an MPC is the mail sorting by type and destination, although it can also work as a point of the course to concentrate and forward mail to other MPCs, where the treatment process takes place.

The mail classification by type is made on a size basis (small, medium and large) or by special service types (express mail and registered mail), being the various types processed in different treatment areas. Each mail type is entirely processed in the same treatment area, even if it includes different delivery speeds.

For some mail types and in some MPCs there are automatic processes to sort by destination. Other mail types (e.g. registered mail) are entirely treated by manual processes.

The mail-objects processed have different characteristics. Each MPC has five TAs (designated by $\left.\mathrm{TA}_{l}, l=1, \ldots, 5\right)$, according to the following features:

- $\mathrm{TA}_{1}$ : large mail-objects, including packages and parcels,

- $\mathrm{TA}_{2}$ : medium mail-objects: essentially periodical publications, like magazines and newspapers,

- $\mathrm{TA}_{3}$ : flats: post-cards and letters up to $20 \mathrm{~g}(0.71 \mathrm{oz})$,

- $\mathrm{TA}_{4}$ : express-mail,

- $\mathrm{TA}_{5}$ : registered mail.

In each TA, mail is treated (sorted and separated) in a chain of specialized treatment units $\left(\mathrm{TU}_{i}\right)$, characterized by manual and automatic operations. This chain defines a productive process, where one or several units feed other units in a well established sequence. A scheme that characterizes an MPC is presented in Fig. 1.

The MPC operates continuously during all the day, although some TAs are closed during a certain period of the day. 


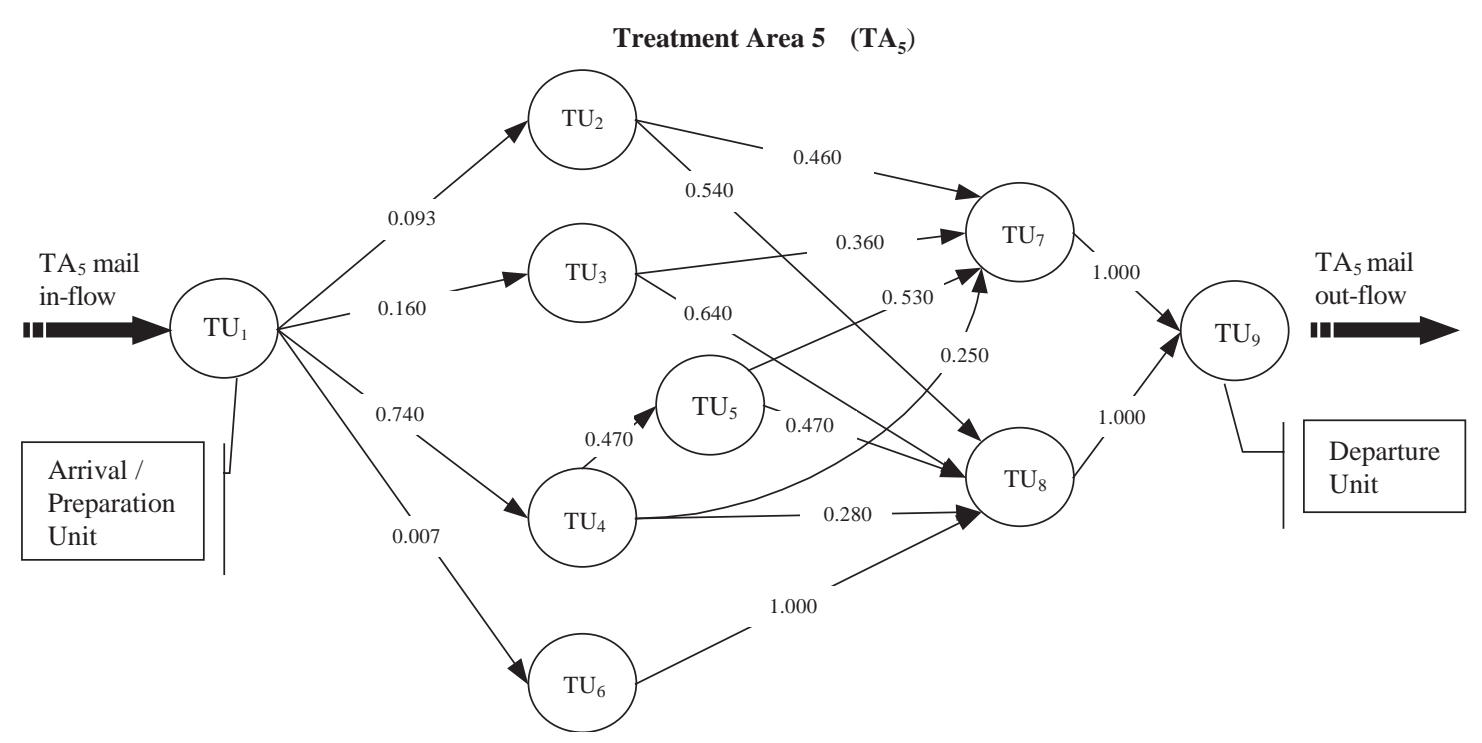

Fig. 2. Scheme of a TA $\left(\mathrm{TA}_{5}\right)$.

There is a chain of treatment units $\left(\mathrm{TU}_{i}\right)$ inside each treatment area, which guarantees mail sorting and separation. Mail objects have to pass through a predefined sequence of TUs, where the percentage of mail-objects that is transferred among each pair of TUs is known. Those transfer rates are estimated from samples drawn on a regular basis, revealing very small changes along the time and for this reason being updated in relatively large periods of time. Notice that these transfer rates depend mainly on the specificity of the operations involved in each TU.

We assume no difficulty in the circulation of mail-objects among TUs and all the mail already fully processed goes to a final expedition unit $\left(\mathrm{TU}_{M}\right)$ in each TA ( $M$ is the total number of TUs).

Mail transfer rates among every pair of TUs is defined in an $(M \times M)$ matrix $P$, where $p_{r i}$ is the transfer rate among $\mathrm{TU}_{r}$ and $\mathrm{TU}_{i}$, and $p_{r i}$ is equal to 0 when there is no connection between that pair of TUs.

Fig. 2 displays the network describing the sequence of treatment units in $\mathrm{TA}_{5}$ (registered mail). The label in each line connecting two units is their corresponding transfer rate. If two units are not connected, they do not transfer any mail between them. $\mathrm{TU}_{1}$ (and other non mentioned TUs) receive all the $\mathrm{TA}_{5}$ incoming mail and $\mathrm{TU}_{9}$ sends all the treated mail to outside. For simplicity, the feed up structure is not represented in the scheme.

The treatment process is made during fixed periods of $15 \mathrm{~min}$. The complete sequence of consecutive periods defines the global plan horizon. Each TA has its own plan horizon, corresponding to their daily working time duration, which might be as long as $24 \mathrm{~h}$. We also define a $1 \mathrm{~h}$ block as a sequence of four consecutive periods, where each block starts on the hour. Fig. 3 presents a graphical illustration of the various time components used in the treatment process.

Fig. 4 describes the mail flow scheme, through the periods, between two general treatment units $\mathrm{TU}_{r}$ and $\mathrm{TU}_{i}$. In each $\mathrm{TU}_{i}$ and at each period $j$, there is an incoming mail quantity which is the sum of the mail directly arrived from outside, the mail at the entrance of $\mathrm{TU}_{i}$ and not treated in 


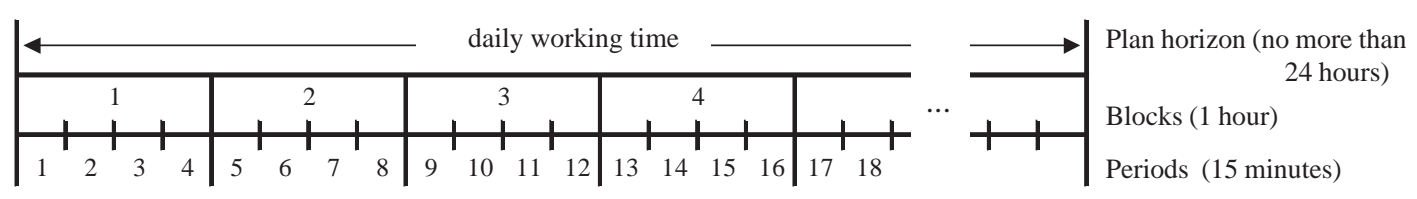

Fig. 3. Graphical illustration of the various time components used in the treatment process.

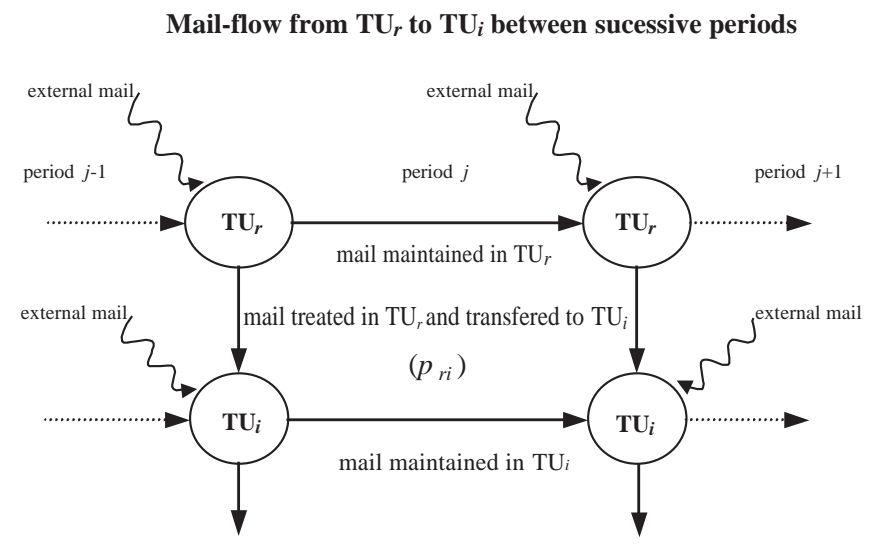

Fig. 4. Mail-flow scheme, through the periods, between two general treatment units $\mathrm{TU}_{r}$ and $\mathrm{TU}_{i}$. The scheme includes external mail entering in each period in both TUs.

the previous periods, and the mail received from preceding units at the end of period $j-1$. This global quantity at the entrance of $\mathrm{TU}_{i}$ in the beginning of period $j$ is divided into mail that is treated during period $j$ and mail that does not leave the entrance of $\mathrm{TU}_{i}$ and waits for its treatment in a future period in that TU.

Each TA receives their corresponding mail during the daily working time. Mail entered in period $j-1$ is ready to start the treatment process in period $j$. The mail arriving instants depend on the closing time of the MDCs and from their distance to the MPC, implying, in general, very small variations in those arriving instants. Hence, the amounts of mail arrived during each individual period is strongly dependent on the global daily mail flow generated at MDCs and not from the particular behaviour of those MDCs. Those daily amounts change along the year, with peak weeks in some periods of the year. Variations on these amounts will be discussed for the particular case study proposed in this paper.

In order to give the chance to any mail-object to go through on the treatment process, each $\mathrm{TU}_{i}$ is not allowed to receive mail $\alpha_{i}$ periods before the end of the plan horizon, where $\alpha_{i}$ defines a certain number of periods and is established by the maximum number of TUs that a mail-object entered from outside to $\mathrm{TU}_{i}$ may need to cover until reaching the last $\mathrm{TU}$. Mail arrived to an already closed TU will be treated on the next day.

An upper limit on the quantity of mail that can remain in each $\mathrm{TU}_{i}$ at the end of the daily working time is assumed. We also impose a standard quality criteria of $99 \%$ on the total volume of mail that must be treated at the end of that same period, i.e. ready to leave the corresponding TA. The remaining $1 \%$ includes mail that is to be treated in the next day. 
The Staff Management Department intends to plan shift scheduling schemes, to face average and extreme scenarios of daily income mail amounts to each individual TA. The working shifts in use in the company range between 3 and $8 \mathrm{~h}$ shifts, corresponding to $3-8$ consecutive $1 \mathrm{~h}$ blocks. All these working shifts must be included in the overall TA daily working time. Each shift has a fixed cost per employee, according to its duration. The purpose of this problem is to minimize the overall workforce salaries, according to the global assignment of employees to each working shift determined.

In each TA, the employees' assignment plan is established for each week. So, the problem is solved prior to each week, planning the workforce needs, according to the amounts of mail expected to be received in that week. This expectation is based on empirical evaluation, based on historical data and on typical seasonal behaviour. In fact, we can observe peak weeks, corresponding to lower and higher income flow amounts. This behaviour varies according to the type of mail considered in each particular TA.

In general, workforce availability inside the MPC can be shared between TAs and used for individual TAs weekly workforce planning. On the other hand, during each week plan established, the employees cannot move from one TA to another. We also assume that any employee can work in any TU inside each TA, at different periods of time. This change between TUs can only occur on the hour, so that in each $1 \mathrm{~h}$ block we have a permanent number of employees in each TU. Out of $\mathrm{TU}_{M}$, where we assume no need for manpower assignment, we consider a limit on the number of employees in each $\mathrm{TU}_{i}$ and in each block.

\section{A mixed integer linear formulation}

In this section, we propose a mixed integer linear formulation for the problem introduced in the previous section. This formulation combines two sets of constraints, namely lotsizing constraints to characterize the batching structure of the problem, and shift scheduling constraints, defining its staff planning structure. As stated before, the objective of this study is concentrated in this last aspect. We start by presenting all the variables, parameters and constraints for the model. A few explanations of the main aspects of the formulation are given next.

\section{Parameters:}

$M \quad$ total number of TUs, ( $\mathrm{TU}_{M}$ is the last unit in the treatment area)

$N$ total number of periods in the time operating interval of the TA

$H \quad$ number of $1 \mathrm{~h}$ blocks

$K \quad$ total number of shifts

$c_{k} \quad$ shift $k$ employees individual salary, $k=1, \ldots, K$

$q_{i j} \quad$ quantity of mail that enters the $\mathrm{TA}$ and directly arrives at $\mathrm{TU}_{i}$ during period $j-1, i=$ $1, \ldots, M-1, j=1, \ldots, N$

$p_{r i} \quad$ transfer rate of mail-objects from $\mathrm{TU}_{r}$ to $\mathrm{TU}_{i}, r, i=1, \ldots, M$ and $r \neq i$

$b_{i} \quad$ quantity of mail-objects that a employee can handle in each period in $\mathrm{TU}_{i}, i=1, \ldots, M-1$

$l_{i} \quad$ upper limit on the number of employees at $\mathrm{TU}_{i}$ in each $1 \mathrm{~h}$ block, $i=1, \ldots, M-1$

$d_{k} \quad$ upper limit on the number of employees in shift $k, k=1, \ldots, K$

$e_{i} \quad$ upper limit on mail quantity allowed to stay in $\mathrm{TU}_{i}$ at the end of the process, $i=1, \ldots, M-1$ 
$a_{k h} \quad 1$ if shift $k$ covers the $h$ th $1 \mathrm{~h}$ block, 0 otherwise, $k=1, \ldots, K, h=1, \ldots, H$

$\alpha_{i} \quad$ number of periods that each $\mathrm{TU}_{i}$ is closed for mail entrance, before the end of the daily working time, $i=1, \ldots, M-1$

\section{Variables:}

$x_{i j}$ nonnegative variable, representing the quantity of mail-objects treated in $\mathrm{TU}_{i}$ during period $j$, $i=1, \ldots, M, j=1, \ldots, N$

$s_{i j}$ nonnegative variable, representing the number of mail-objects kept at the end of period $j$ at $\mathrm{TU}_{i}, i=1, \ldots, M, j=1, \ldots, N$

$w_{i h}$ integer variable, defining the number of employees in $\mathrm{TU}_{i}$ during block $h, i=1, \ldots, M-1, h=$ $1, \ldots, H$

$v_{k} \quad$ integer variable, defining the number of employees assigned to shift $k, k=1, \ldots, K$

In this formulation we consider $x_{i 0}=0$ and $s_{i 0}=0(i=1, \ldots, M), x_{M j}=0(j=1, \ldots, N)$ and $w_{M h}=0(h=1, \ldots, H)$; so we can ignore those variables from the model. In order to guarantee the treatment of the majority of mail-objects that enters the TA, we close each $\mathrm{TU}_{i}$ entrance of mail at the $\left(N-\alpha_{i}\right)$ th period, considering $\alpha_{i}$ as the maximum number of TUs that a mail-object entered from outside to $\mathrm{TU}_{i}$ may need to cover until reaching $\mathrm{TU}_{M}$. This means that all $q_{i j}$, for $i=1, \ldots, M$ and $j=N-\alpha_{i}+1, \ldots, N$, should be ignored from the model. The same happens to $q_{M j}$, because no mail arrives directly to $\mathrm{TU}_{M}$.

\section{Formulation 1:}

$$
\begin{array}{ll}
\text { Minimize } & \sum_{k=1}^{K} c_{k} v_{k} \\
\text { Subject to } & q_{i j}+s_{i, j-1}+\sum_{\substack{r=1 \\
r \neq i}}^{M-1}\left(p_{r i} x_{r, j-1}\right)=x_{i j}+s_{i j}, \quad i=1, \ldots, M, \quad j=1, \ldots, N, \\
& s_{i N}+\sum_{r=1}^{M-1} p_{r i} x_{r N} \leqslant e_{i}, \quad i=1, \ldots, M-1, \\
& s_{M N}+\sum_{r=1}^{M-1} p_{r M} x_{r N} \geqslant 0.99 \sum_{i=1}^{M-1} \sum_{j=1}^{N-\alpha_{i}} q_{i j} \\
& x_{i j} \leqslant b_{i} w_{i h}, \quad i=1, \ldots, M-1, j=1, \ldots, N, h=\lceil j / 4\rceil, \\
& w_{i h} \leqslant l_{i}, \quad i=1, \ldots, M-1, \quad h=1, \ldots, H, \\
& \sum_{i=1} w_{i h}=\sum_{k=1}^{K} a_{k h} v_{k}, \quad h=1, \ldots, H, \\
& v_{k} \leqslant d_{k}, \quad k=1, \ldots, K,
\end{array}
$$




$$
\begin{aligned}
& x_{i j} \geqslant 0, \quad i=1, \ldots, M, \quad j=1, \ldots, N, \\
& s_{i j} \geqslant 0, \quad i=1, \ldots, M, \quad j=1, \ldots, N, \\
& w_{i h} \in N_{0}, \quad i=1, \ldots, M-1, \quad h=1, \ldots, H, \\
& v_{k} \in N_{0}, \quad k=1, \ldots, K .
\end{aligned}
$$

Constraints (2) are the flow conservation constraints in each $\mathrm{TU}_{i}$ and in each period $j$, and they establish the equilibrium between the incoming and the outgoing mail quantity in the mentioned TU in period $j$. The incoming mail is defined as the sum of the mail arrived during the $(j-1)$ th period $\left(q_{i j}\right)$, the mail at the entrance of $\mathrm{TU}_{i}$ and not treated in the previous periods $\left(s_{i, j-1}\right)$, and the mail received from preceding units at the end of period $j-1\left(\sum_{r=1 r \neq i}^{M} p_{r i} x_{r, j=1}\right)$. This quantity at the entrance of $\mathrm{TU}_{i}$ in the beginning of period $j$ must be equal to the mail treated in period $j\left(x_{i j}\right)$ plus the mail that does not leave the entrance of $\mathrm{TU}_{i}\left(s_{i j}\right)$ and waits to be treated in a future period in $\mathrm{TU}_{i}$. Constraints (3) impose an upper limit on the number of mail-objects allowed to stay in each TU at the end of the process. The $\left(p_{r i} x_{r N}\right)$ terms represent mail treated in preceding TUs, which are transferred to $\mathrm{TU}_{i}$ at the end of the $N$ th period. Inequality (4) guarantees that $99 \%$ of the overall mail that enters in TA is assembled in the final $\mathrm{TU}_{i}$ at the end of the process. Notice that $x_{M j}=0(j=1, \ldots, N)$, so the final arrival TU simply accumulates (almost) all the mail flow and it does not require any employees. These three sets of constraints characterize the multi-level lotsizing structure of the problem. On the other hand, inequalities (6)-(8) describe the shift scheduling subproblem. Considering this separation, inequalities (5) can be seen as coupling constraints, relating the treatment variables $\left(x_{i j}\right)$ with the staff slot variables $\left(w_{i h}\right)$. They guarantee a sufficient number of employees to cover the mail treatment in each period in $\mathrm{TU}_{i}$. Constraints (6) and (8) impose upper limits to the number of employees in each $\mathrm{TU}_{i}$ and in each shift $k$, respectively. We relate these two sets of variables ( $w_{i h}$ and $v_{k}$ ) in constraints (7), guaranteeing the employees assignment among shifts and their corresponding $1 \mathrm{~h}$ blocks, according to the various shift intervals allowed. The objective is to minimize the overall workforce cost in this TA, which is stated in the objective function (1).

We consider variables $x_{i j}$ and $s_{i j}$ as nonnegative, although representing nondivisible units (mailobjects). This option simplifies the problem and represents a negligible lost to the correct description of the problem, due to the discrepancy of a unit in the total amount of mail treated. The other variables represent employees and have to be defined as integer variables.

An interesting aspect of this model is characterized by the coupling constraints (7). Contrary to a staff decision problem, we do not simply impose a cover from a selection of shifts over the total workforce in each one hour block, defined by the inequalities $\sum_{i=1}^{M-1} w_{i h} \leqslant \sum_{k=1}^{K} a_{k h} v_{k}$ to each $h \in\{1, \ldots, H\}$. Instead, we further demand the two quantities in each term to be equal, which implies Eqs. (7). The solutions to be dropped (those verifying $\sum_{i=1}^{M-1} w_{i h}<\sum_{k=1}^{K} a_{k h} v_{k}$ ), are concerned with cases where we have a smaller number of employees assigned to TUs than those assigned to the shifts covering a certain block $h$. We can show that any of those solutions can be substituted by another feasible solution, obtained from the previous one by just increasing the values of the variables $w_{i h}$ until we reach the equality defined by constraint (7). Both solutions have the same objective function value. By using the equality constraints a significant number of dominated solutions are dropped from 
the model. This reduction is especially important in the linear relaxation version of the model, which may contribute to the efficiency of the optimization process, when LP relaxation techniques are used to solve the problem. This is highlighted in Chapter 5 which reports computational experience with this type of models.

An important aspect on the mentioned transformation is related with the assurance of its feasibility, according to the upper-bound limit imposed to the number of employees in each TU, defined in constraints (6). In this case, by adding constraints (6) to every $i \in\{1, \ldots, M-1\}$, we obtain the following fixed upper limit $\sum_{i=1}^{M-1} w_{i h} \leqslant \sum_{i=1}^{M-1} l_{i}=L$, which is the same in every $1 \mathrm{~h} \mathrm{block.} \mathrm{Hence}$ $\sum_{k=1}^{K} a_{k h} v_{k} \leqslant L$, so for two different blocks $h_{1}$ and $h_{2}$, the upper bound defined in constraints (6) do not impose a positive gap between $\sum_{i=1}^{M-1} w_{i h_{1}}$ and $\sum_{k=1}^{K} a_{k h_{1}} v_{k}$. This allows at the same time an equality between the same two terms in block $h_{2}$, as both $\sum_{k=1}^{K} a_{k h_{1}} v_{k}$ and $\sum_{k=1}^{K} a_{k h_{2}} v_{k}$ have the same upper bound $(L)$. This means that the upper limit constraints (6) neither exclude the representative valid solutions from the model, nor turn the problem to be infeasible.

\section{A case study: the treatment area $\mathrm{TA}_{5}$}

The formulation proposed in Section 3 characterizes a general operating structure of a TA. In this section, we concentrate our attention in a specific treatment area, namely $\mathrm{TA}_{5}$, in which registered mail is treated. Next, some particular features related with this area are included, which follow from the network presented in Fig. 2. The daily working time in this particular case is defined from 17:00 to $04: 00$. Table 1 includes the corresponding working shifts.

Two distinct time intervals are considered in the overall daily working time. The first starts at 17:00 and ends at 23:00, while the second one starts immediately after the end of the first and ends at 4:00. We refer to both time intervals as working time 1 and 2, respectively. Some characteristics of both periods are stated below.

Time interval 1-from 17:00 to 23:00: In this time interval, regional mail (addressed to the same MPC area) arrives at $\mathrm{TA}_{5}$ together with mail to other MPC areas (inter-regional mail). So it is

Table 1

Feasible working shifts in $\mathrm{TA}_{5}$, ordered sequentially

Working shifts

\begin{tabular}{lllllllllllll}
\hline \multicolumn{2}{l}{ No. $3 \mathrm{~h}$} & \multicolumn{2}{l}{ No. $4 \mathrm{~h}$} & \multicolumn{2}{l}{ No. $5 \mathrm{~h}$} & \multicolumn{2}{l}{ No. $6 \mathrm{~h}$} & \multicolumn{2}{l}{ No. $7 \mathrm{~h}$} & \multicolumn{2}{l}{ No. $8 \mathrm{~h}$} \\
\hline 1 & {$[17: 00-20: 00]$} & 10 & {$[17: 00-21: 00]$} & 18 & {$[17: 00-22: 00]$} & 25 & {$[17: 00-23: 00]$} & 31 & {$[17: 00-24: 00]$} & 36 & {$[17: 00-01: 00]$} \\
2 & {$[18: 00-21: 00]$} & 11 & {$[18: 00-22: 00]$} & 19 & {$[18: 00-23: 00]$} & 26 & {$[18: 00-24: 00]$} & 32 & {$[18: 00-01: 00]$} & 37 & {$[18: 00-02: 00]$} \\
3 & {$[19: 00-22: 00]$} & 12 & {$[19: 00-23: 00]$} & 20 & {$[19: 00-24: 00]$} & 27 & {$[19: 00-01: 00]$} & 33 & {$[19: 00-02: 00]$} & 38 & {$[19: 00-03: 00]$} \\
4 & {$[20: 00-23: 00]$} & 13 & {$[20: 00-24: 00]$} & 21 & {$[20: 00-01: 00]$} & 28 & {$[20: 00-02: 00]$} & 34 & {$[20: 00-03: 00]$} & 39 & {$[20: 00-04: 00]$} \\
5 & {$[21: 00-24: 00]$} & 14 & {$[21: 00-01: 00]$} & 22 & {$[21: 00-02: 00]$} & 29 & {$[21: 00-03: 00]$} & 35 & {$[21: 00-04: 00]$} & \\
6 & {$[22: 00-01: 00]$} & 15 & {$[22: 00-02: 00]$} & 23 & {$[22: 00-03: 00]$} & 30 & {$[22: 00-04: 00]$} & & & \\
7 & {$[23: 00-02: 00]$} & 16 & {$[23: 00-03: 00]$} & 24 & {$[23: 00-04: 00]$} & & & & & \\
8 & {$[00: 00-03: 00]$} & 17 & {$[00: 00-04: 00]$} & & & & & & & & \\
9 & {$[01: 00-04: 00]$} & & & & & & & & & & & \\
\hline
\end{tabular}


necessary to separate both types of mail and to sort them by destination. There are two demands in the treatment of the mail:

(i) $\mathrm{TU}_{7}$ simply treats inter-regional mail, which has to be ready for expedition before 23:00. This means that inter-regional mail (which comes from $\mathrm{TU}_{7}$ ) should be ready in $\mathrm{TU}_{9}$ before the end of the first time interval. The regional mail processed during this time interval is kept at $\mathrm{TU}_{8}$. Although there are two different items (regional and inter-regional mail) in this problem, we consider it as a single-item case.

(ii) Regional mail must be treated before 04:00 and should be in $\mathrm{TU}_{9}$ before that time.

Time interval 2-from 23:00 to 04:00: In this period only regional mail (addressed to the same MPC area) arrives at $\mathrm{TA}_{5}$. This mail does not require any kind of preparation, hence node 1 is eliminated from the network.

Therefore, the original matrix $P=\left[p_{r i}\right]_{r, i=1, \ldots, M}$ is splitted into two matrices $P 1=\left[p 1_{r i}\right]_{r, i=1, \ldots, M}$ and $P 2=\left[p 2_{r i}\right]_{r, i=1, \ldots, M}$, where $M$ is the number of TUs and $P 1$ and $P 2$ are concerned with the time intervals 1 and 2 , respectively.

$\mathrm{TU}_{M-2}$ and $\mathrm{TU}_{M-1}$ can share their human resources availability, so we consider their manpower assignment in conjunction. We simply demand mail to be kept separated (regional and inter-regional), while treated by the same team.

Considering these further characteristics, the following mixed integer programming model can be associated to the treatment area $\mathrm{TA}_{5}$, where $N 1$ represents the number of periods until 23:00.

\section{Formulation 2:}

Minimize $\sum_{k=1}^{K} c_{k} v_{k}$

$$
\begin{aligned}
& \text { Subject to } q_{i j}+s_{i j-1}+\sum_{\substack{r=1 \\
r \neq i}}^{M-1}\left(p 1_{r i} x_{r j-1}\right)=x_{i j}+s_{i j}, \quad i=1, \ldots, M, \quad j=1, \ldots, N 1 \text {, } \\
& q_{i j}+s_{i j-1}+\sum_{\substack{r=1 \\
r \neq i}}^{M-1}\left(p 2_{r i} x_{r j-1}\right)=x_{i j}+s_{i j}, \quad i=1, \ldots, M, \quad j=N 1+1, \ldots, N, \\
& s_{i N 1}+\sum_{\substack{r=1 \\
r \neq i}}^{M-1} p 1_{r i} x_{r N 1} \leqslant 0.01\left(\sum_{j=1}^{N 1-\alpha_{i}} q_{i j}+\sum_{j=1}^{N 1} \sum_{\substack{r=1 \\
r \neq i}}^{M-1} p 1_{r i} x_{r j}\right), \\
& i=1, \ldots, M-2 \text {, } \\
& s_{i N}+\sum_{\substack{r=1 \\
r \neq i}}^{M-1} p 2_{r i} x_{r N} \leqslant 0.01\left(\sum_{j=1}^{N-\alpha_{i}} q_{i j}+\sum_{j=1}^{N 1} \sum_{\substack{r=1 \\
r \neq i}}^{M-1} p 1_{r i} x_{r j}+\sum_{j=N 1+1}^{N} \sum_{\substack{r=1 \\
r \neq i}}^{M-1} P 2_{r i} x_{r j}\right), \\
& i=1, \ldots, M-1 \text {, }
\end{aligned}
$$




$$
\begin{aligned}
& \sum_{i=M-1}^{M}\left(s_{i N 1}+\sum_{\substack{r=1 \\
r \neq i}}^{M-1} p 1_{r i} x_{r N 1}\right) \geqslant 0.99 \sum_{i=1}^{M-1} \sum_{j=1}^{N 1-\alpha_{i}} q_{i j}, \\
& s_{M N} \sum_{\substack{r=1 \\
r \neq i}}^{M-1} P 2_{r M} x_{r N} \geqslant 0.99 \sum_{i=1}^{M-1} \sum_{j=1}^{N-\alpha_{i}} q_{i j}, \\
& x_{i j} \leqslant b_{i} w_{i h}, \quad i=1, \ldots, M-3, \quad j=1, \ldots, N, \quad h=\lceil j / 4\rceil, \\
& x_{M-2 j}+x_{M-1 j} \leqslant b_{M-2} w_{M-2 h}, \quad j=1, \ldots, N, \quad h=\lceil j / 4\rceil, \\
& w_{i h} \leqslant l_{i}, \quad i=1, \ldots, M-2, \quad h=1, \ldots, H, \\
& \sum_{i=1}^{M-2} w_{i h}=\sum_{k=1}^{K} a_{k h} v_{k}, \quad h=1, \ldots, H, \\
& x_{i j} \geqslant 0, \quad i=1, \ldots, M, \quad j=1, \ldots, N, \\
& s_{i j} \geqslant 0, \quad i=1, \ldots, M, \quad j=1, \ldots, N, \\
& w_{i h} \in N_{0}, \quad i=1, \ldots, M-2, \quad h=1, \ldots, H, \\
& v_{k} \in N_{0}, \quad k=1, \ldots, K .
\end{aligned}
$$

As before, variables $x_{i 0}, s_{i 0}(i=1, \ldots, M)$ and $x_{M, j}(j=1, \ldots, N)$ and parameters $q_{i j}(i=1, \ldots, M$; $\left.j=N-\alpha_{i}+1, \ldots, N\right)$ can be ignored from the model. Furthermore, it follows from the network presented in Fig. 2 that there are $M=9$ TU units and $\alpha_{1}=4, \alpha_{4}=3, \alpha_{2}=\alpha_{3}=\alpha_{5}=\alpha_{6}=2, \alpha_{7}=\alpha_{8}=1$. Remember that $\mathrm{TU}_{9}$ does not receive mail from outside.

This formulation differs from the previous one in some constraints, that are discussed next. Those differences are directly related with the mentioned features of the treatment area $\mathrm{TA}_{5}$. In the set of flow conservation constraints (11a) and (11b), matrices $P 1$ and $P 2$ define the new transfer rate network structure in both time intervals. Constraints (12a) and (12b) substitute inequalities (3), which define upper limits to the end process residual number of mail-objects that can be left in each TU and are directly dependent on the global volume of mail arrived to each of those TUs (no more than $1 \%$ of this quantity), in both end time periods, $N 1$ and $N$, respectively. The new constraints establish a non constant upper limit on the amount of mail allowed to remain in each TU, differing from inequalities (3) proposed for the general case. Out of the $99 \%$ of mail to be treated at the end of the process and defined by the constraint (13b), we must guarantee that all the mail entered until the $\left(N 1-\alpha_{i}\right)$ th period should be at $\mathrm{TU}_{M-1}$ (regional mail) and at $\mathrm{TU}_{M}$ (inter-regional mail) until the end of period $N 1$. This is defined in the constraint (13a), allowing a $1 \%$ tolerance of unsent inter-regional mail plus non accumulated regional mail at $\mathrm{TU}_{M-1}$, at the end of the $N 1$ th period. Notice that this $1 \%$ tolerance of undelivered mail is established by the standard quality criteria, while the previous $1 \%$ TUs residual mail tolerance is an internal organizational criteria, so both concepts cannot be self-substituted. The lower limit on the number of employees $\left(w_{i h}\right)$ imposed by the amount of mail treated $\left(x_{i j}\right)$ is the same as before. We consider apart the $\mathrm{TU}_{M-2}$ and $\mathrm{TU}_{M-1}$ cases, where 
both units amount of mail treated is covered by the number of employees assigned to $\mathrm{TU}_{M-2}$. This leads into constraints (14b). Notice that there is no need of human resources assigned to $\mathrm{TU}_{M}$. As mentioned at the end of Section 2.2, workforce availability inside an MPC can be shared among TAs and used for individual TAs weekly workforce planning. TA 5 is not very demanding in human resources needs, when compared with other TAs. For this reason, no upper limits to the number of employees in each shift are assumed, hence inequalities (8) have be deleted from the present model.

\section{Computational experiments to the case study}

Consider the case study presented in the previous section and based in Fig. 2 treatment scheme. Furthermore, let the daily working time of $\mathrm{TA}_{5}$ be the one settled before, from 17:00 to 4:00. Hence, the following parameters are set in Formulation 2:

(i) $M=9$-number of TUs,

$N=44$-number of periods $(N 1=24)$,

$H=11$-there are 11 blocks in the 17:00 to 04:00 time interval,

$K=39$-number of working shifts of 3-8 h, as defined in Table 1 .

(ii) Costs for each working shift of $3,4,5,6,7$ and $8 \mathrm{~h}:\left(c_{k}, k=1, \ldots, K\right)$

\begin{tabular}{lllllll}
\hline Shifts duration $(\mathrm{h})$ & 3 & 4 & 5 & 6 & 7 & 8 \\
\hline Cost $(€)$ & 18.66 & 24.88 & 31.10 & 37.32 & 43.54 & 49.76
\end{tabular}

All the costs are proportional to $6.22 €$, defining the hourly cost of work. Nonproportional costs, according to shifts duration, do not impose any further difficulties to the problem.

(iii) Matrices $P 1$ and $P 2$ are defined in Appendix A, where $p 1_{r i}$ is the mail-flow transfer rate from $\mathrm{TU}_{r}$ to $\mathrm{TU}_{i}(r, i=1, \ldots, M)$ in the 17:00-23:00 time interval, and $p 2_{r i}$ is the mail-flow transfer rate from $\mathrm{TU}_{r}$ to $\mathrm{TU}_{i}(r, i=1, \ldots, M)$ in the 23:00-04:00 time interval.

(iv) The parameters $q_{i j}(i=1, \ldots, M-1, j=1, \ldots, N)$ are defined in Appendix A, representing the number of mail-objects arrived directly to each TU per period.

(v) We also consider processing speed rate values, defined by $b_{i}(i=1, \ldots, M)$ parameters. These are presented in the following table, by mail-objects/man per period:

\begin{tabular}{llllllll}
\hline TU & 1 & 2 & 3 & 4 & 5 & 6 & 7 and 8 \\
\hline Mail-objects/man & 1500 & 250 & 280 & 313 & 313 & 25 & 350 \\
\hline
\end{tabular}

We assume that no more than 15 employees can simultaneously be in the same TU, so $l_{i}=15$ for $i=1, \ldots, M$. As stated before, we have ignored the upper limit on the number of employees on the same working shift, which means that constraints (8) have been dropped from the model.

We have used the ILOG/CPLEX 7.0 package LP and mixed integer (MIP) solver to process the mixed integer linear programming Formulation 2 with all the mentioned parameters. The branch-and-cut version of the MIP solver has been chosen, which allows the generation of 
general feasible cuts, provided by the software. The optimization model has been run in a Pentium III $800 \mathrm{MHz}$ processor machine.

Next some scenarios are analysed, showing the problem sensitivity to some of its input parameters. These changes focus on the daily amount of mail arrived to $\mathrm{TA}_{5}$ and on the quality criteria level. As mentioned in Section 2.2, the mail arriving instants to this area, and also to the other ones, depend on the closing time of the MDCs and on their distance to the MPC, implying, in general, very small variations in those arriving instants. Hence, the amounts of mail arrived during each individual period are strongly dependent on the global daily mail flow generated at MDCs and not to the particular behaviour of those MDCs. Those daily amounts change along the year, with peak weeks in some periods of the year. In this paper, year 2001 daily income mail-flow is analysed for $\mathrm{TA}_{5}$, in order to evaluate human resources needs from lower to higher amounts of mail entered in that specific TA. Those observations are important to plan future manpower needs at $\mathrm{TA}_{5}$.

Consider the total amount of mail arrived to $\mathrm{TA}_{5}$ in a specific day, defined by $Q=\sum_{i=1}^{M-1} \sum_{j=1}^{N-\alpha_{i}} q_{i j}$. Then, for the year 2001, the 251 daily observed $Q$ values conduct to the following statistical parameters: $\bar{x}=46925, s=13715.923$, skewness $=-0.29$ (with std. error of skewness $=0.148$ ) and kurtosis $=0.458$ (with std. error of kurtosis $=0.148$ ). Although both quotients (skewness and kurtosis divided by their respective std. errors) fall between -2 and 2, the Kolmogorov-Smirnov (with Lillierfors correction) goodness-of-fit test gives no evidence that the sample comes from the Normal distribution with the estimated sample parameters, presenting a $p$-value equal to 0.002 . We used the SPSS statistical software, version 11.0 (see [40]).

Based on these observations, the following four points are to be addressed next:

(i) the average case $(Q=46925)$, including some aspects related with Formulation 2 resolution and some changes on the model parameters;

(ii) a comparison between small, average and high mail amount cases, considering $Q=36709$ (first quartile), $Q=46925$ and $Q=55006$ (third quartile), respectively;

(iii) the analysis of the weeks with small and high average amounts of mail arrived, considering the median observation in those weeks, with $Q=25947$ and $Q=69039$, respectively;

(iv) the analysis of the weeks with highest and shortest differences between the maximum and the median observations.

Due to some privacy options of the Company, the comparison with the practical solutions in use are simply provided for the aggregated average case.

The $q_{i j}$ parameters table, presented in Appendix A, defines the average case income mail quantities arrived per period, so for $Q=46925$. For different $Q$ values, the $q_{i j}$ quantities are proportional to those presented in the mentioned table.

(i) Average case: The linear relaxation optimal solution value of Formulation 2 defines a lower bound equal to $610.36 €$, and was obtained after $0.28 \mathrm{~s}$ of CPU time.

The MIP solver of CPLEX 7.0 was unable to reach optimality after $12 \mathrm{~h}$ of running time. Nevertheless, some interesting feasible solutions have been obtained during the branch-and-bound execution. The best lower and upper bounds found at the end of this experience are $638.69 €$ and $646.88 €$, respectively. Hence there is a duality gap of $1.27 \%$. In the first node of the branch-and-bound tree, the MIP solver strength the model with 84 Gomory fractional cuts and four MIR cuts, leading the lower bound to $615.05 €$. After some experiences on node and variable selection strategies of the 


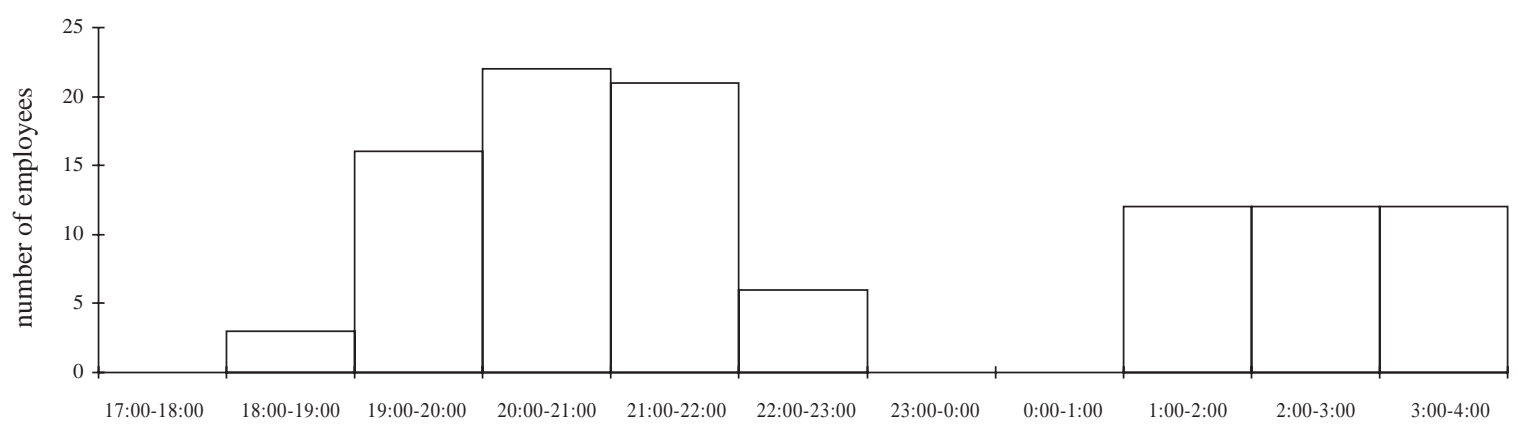

Fig. 5. Number of employees determined per block.

MIP solver, we have chosen to use the best-bound search strategy for node selection and the variable selection based on pseudo-costs (see [39]).

In this case, the normal daily treatment process of this TA is guaranteed by a $104 \mathrm{~h}$ workforce solution, corresponding to a $646.88 €$ daily cost. The $1.27 \%$ duality gap simply corresponds to $1 \mathrm{~h}$ workforce, that is, either the best feasible solution is optimal or there is an optimal solution requiring one less hour.

We have also tried to solve the problem starting the branch-and-bound method from Formulation 2 with constraints (7) defined as less or equal inequalities, characterizing the shifts covering over the hourly assignment of employees to TUs. In this case, we reached the same $104 \mathrm{~h}$ feasible solution, although the lower bound obtained after the same $12 \mathrm{~h}$ of running time was equal to $636.41 €$, defining a $1.62 \%$ duality gap, that is superior to the one obtained in the previous case.

The best feasible solution found is presented in Table 10 in Appendix B, which presents the number of employees determined per block and assigned to each TU: variables $w_{i h}$ values.

Fig. 5 shows the total number of employees per block, according to the $104 \mathrm{~h}$ solution found.

Fig. 6 presents the amount of mail-objects in the system and not yet treated (line) per period $j(j=1, \ldots, 44)$, defined by the difference between the total amount entered before period $j$ and the quantity already arrived at $\mathrm{TU}_{9}$. The figure also shows the total amounts entered per period $j$ (stars) (arrived during the $(j-1)$ th period).

We cannot establish a fair comparison between both charts, because the speed rate (in mail-objects per period) among TUs is different. In any case, if we try to lean Fig. 6 against Fig. 5, it gives an idea of how workforce adapts to the mail amount being treated, per block. That comparison shows, for instance, that the 12 employees assigned to the $3 \mathrm{~h}$ shift (1:00-4:00), which allows for a significant decrease of mail-objects in the (1:00-2:00) block, may reflect some spare capacity in manpower usage in the time interval from 2:00 to 4:00. This spare capacity might be appropriately used if an augmented amount of mail (namely regional mail) arrives to this TA. Notice that our minimum duration shifts are three hours time intervals.

Table 2 presents the number of employees determined per shift type, and described by the solution values of the variables $v_{k}$. No employees have been assigned to the remaining shifts that are not included in this table.

We may think in many alternative scenarios, still centred in the average arrived amount case $(Q=46925)$. Some of these alternative cases are discussed in the next paragraphs, obtained through appropriate changes in some parameters. 


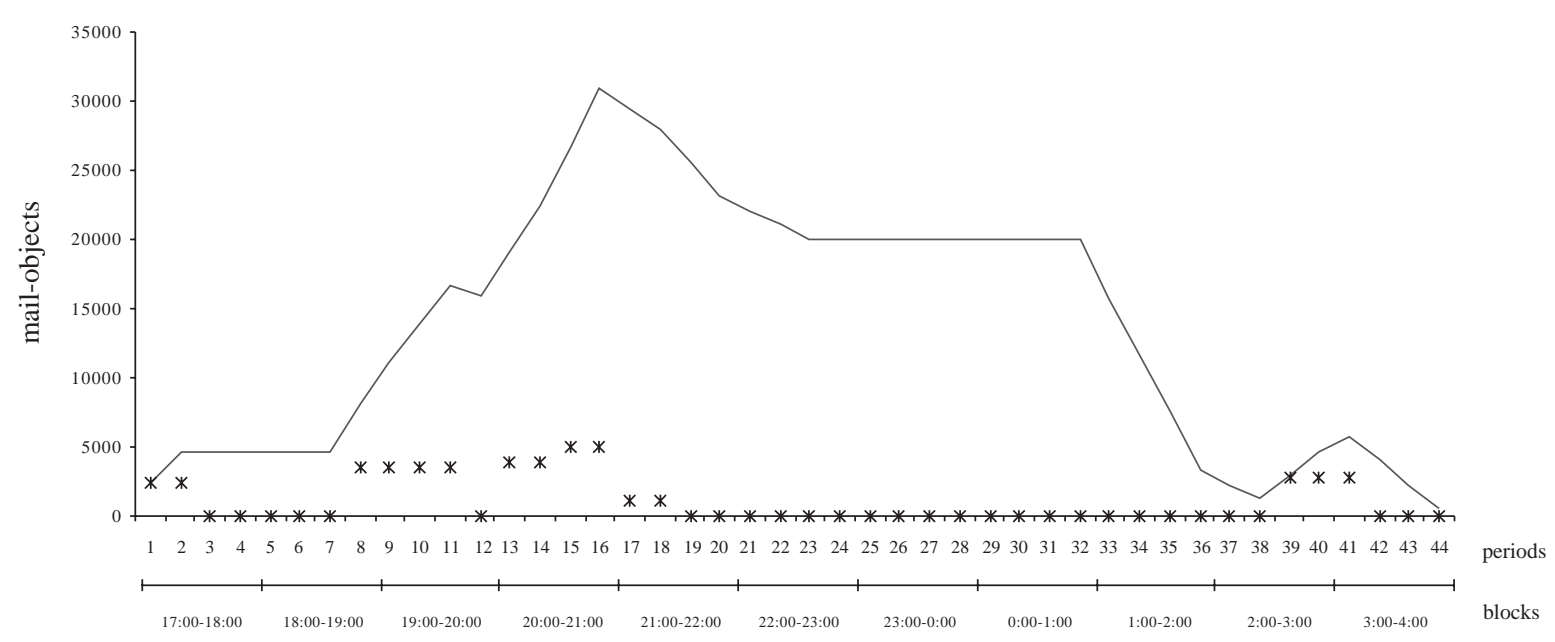

Fig. 6. Amount of mail-objects in the system and not yet treated (line) per period $j$, and the total amounts entered per period $j$ (stars) (arrived during the $(j-1)$ th period), for $j=1, \ldots, 44$.

Table 2

Number of employees determined per shift type: variables $v_{k}$ solution values

\begin{tabular}{lc}
\hline Shifts & Number of employees \\
\hline $18: 00-21: 00$ & 1 \\
$18: 00-22: 00$ & 2 \\
$19: 00-22: 00$ & 13 \\
$20: 00-23: 00$ & 6 \\
$01: 00-04: 00$ & 12 \\
\hline
\end{tabular}

It can be showed that for the same amount of mail-objects arrived $(Q=46925)$, the $104 \mathrm{~h}$ workforce can treat up to 46742 mail-objects, corresponding to $99.61 \%$ of the total amount entered. This means that the proposed workforce can go beyond the $99 \%$ standard for the quality criteria. Notice that the previous reported best feasible solution, described in Table 10 in Appendix B, only treat 46456 mail-objects, corresponding to $99 \%$ of the total amount. The $99.61 \%$ solution has been obtained from a changed version of Formulation 2. The new model involves the maximization of the nonnegative variable $\phi$, representing the standard quality criteria, also appearing in constraints (13a) and (13b). The model includes the following additional constraint:

$$
\sum_{k=1}^{K} \delta_{k} v_{k}=104
$$

to force the use of only $104 \mathrm{~h}$ of workforce ( $\delta_{k}$ is the time duration, in hours, of shift $k$ ).

In a different perspective, we also looked for the maximum increase on the total amount of mail that $\mathrm{TA}_{5}$ can receive, keeping the same $99 \%$ quality criteria and forcing again for a $104 \mathrm{~h}$ of manpower usage. This case involves a different changed version of Formulation 2, which includes a 
Table 3

MIP solver information related with the three quality criteria scenarios: $97 \%, 95 \%$ and $90 \%$

\begin{tabular}{llll}
\hline Quality criteria $(\%)$ & Lower bound $(€)$ & Upper bound & Duality gap $(\%)$ \\
\hline 97 & 617.25 & $628.25 €=101 \mathrm{~h}$ & 1.751 \\
95 & 609.55 & $622.04 €=100 \mathrm{~h}$ & 2.008 \\
90 & 591.85 & $603.38 €=97 \mathrm{~h}$ & 1.911 \\
\hline
\end{tabular}

new nonnegative variable $\beta$, defining the proportional increase in the previous amount of 46925 mail-objects. So the new formulation involves the maximization of $\beta$, subject to Formulation 2 constraints, added by equality (15) and substituting all the parameters $q_{i j}$ by $q_{i j} \beta$, in constraints (11a)-(13b). After $12 \mathrm{~h}$ of the branch-and-bound execution, the best feasible solution found was $\beta=1.0044$, with an upper bound defined by $\beta=1.0258$. This means that we can treat at least 47131 mail-objects with the same workforce resources with no damage to the service quality standards, corresponding to more 206 mail-objects when compared with the initial case (for proportional increases). This increase can not exceed 1210 mail-objects, considering the upper bound value obtained. Remember that it is assumed that an increase on the daily mail amount entered is established by proportional increases in each period mail amounts arrived, according to the $q_{i j}$ values presented in Appendix A.

Another interesting aspect is related with the $99 \%$ standard quality criteria on the total amount of mail treated, and the $1 \%$ of mail that is allowed to stay in each TU and treated in the next day. As mentioned in Section 2.1, an independent sector regulator follows the time series movement of delayed mail. Those limits are agreed with the Company and are frequently a matter of discussion. At this point, we may discuss two scenarios: the $100 \%$ quality criteria; and relaxed standard quality criteria's. We start by the first scenario.

From an optimizer perspective, these tolerances seem to be an imperfection of the system, which should not be assumed by the model. From the manager perspective, these tolerance limits have long been studied, and reflect a good compromise between an admissible negligible failure and the additional workforce cost needed to accomplish this failure. If we try to answer the optimizer expectations by solving Formulation 2 with $100 \%$ quality criteria and $0 \%$ mail left on TUs (providing the appropriate changes in constraints (12a), (12b), (13a) and (13b)), we obtain (again after $12 \mathrm{~h}$ of the branch-and-bound execution) a lower bound equal to $676.43 €$ and an upper bound equal to $690.46 €$. This means that the optimal solution to that specific problem involves between 109 and 111 workforce hours. So to close this residual volume of nontreated mail, we must spend at least more $5 \mathrm{~h}$, representing an increase on the overall manpower effort. This solution is interesting to compare with the previous reported case, which showed that we are able to treat $99.61 \%$ of the global amount entered using only the $104 \mathrm{~h}$ workforce. So the mentioned five additional hours required to meet the $100 \%$ case reflect only $0.39 \%$ (183 mail-objects), when keeping the same global amount entered (46925 mail-objects).

For the relaxed scenarios, we have considered the following standard quality criteria parameters: $97 \%, 95 \%$ and $90 \%$, keeping the $1 \%$ residual mail tolerance at TUs and all the other initial parameters. In this case, the changes are only present in constraints (13a) and (13b). After $12 \mathrm{~h}$ of running time, the MIP solver gave the solutions presented in Table 3. 


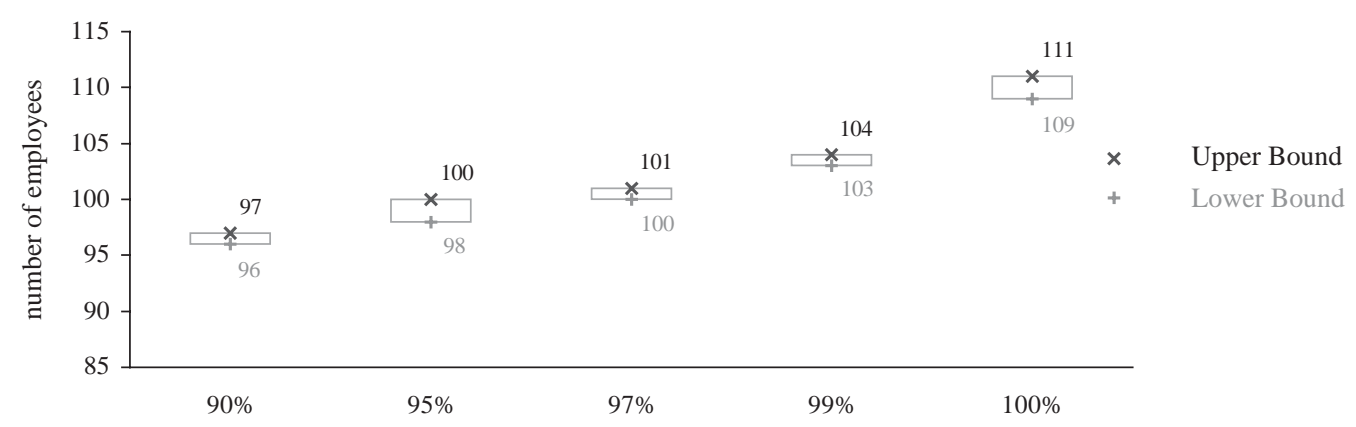

Fig. 7. Lower and upper bound number of employees determined to the five quality criteria scenarios: $90 \%, 95 \%, 97 \%$, $99 \%$ and $100 \%$.

These three scenarios and the previous $99 \%$ and $100 \%$ cases are compared in Fig. 7. All the cases are defined in workforce hours, including the lower bound transformed values, which are ob-

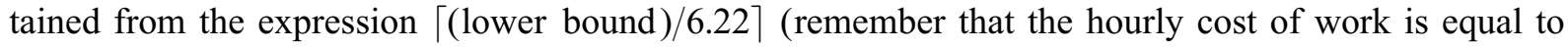
$6.22 €)$, defining a lower limit on manpower usage.

Fig. 7 shows that, when placed at the $99 \%$ case, the additional workforce effort needed to increase the quality criteria is much superior than the benefit obtained from relaxing that same quality criteria. In fact, comparing only the upper bound values, the 1\% quality criteria increase over the $99 \%$ case requires seven additional workforce hours, which coincides with the manpower spared from relaxing the quality criteria to $90 \%$. This subject is a frequent theme of discussion in the Company, to which the previous analysis indicate that a decrease in the delivery quality standards may not correspond to a significant decrease in manpower usage.

In order to compare with the practice in this $\mathrm{TA}_{5}$, we applied to the MPC manager for a practical workforce solution to the 46925 mail-objects case, keeping all the parameters initially introduced. This MPC has recently been installed and the current manager has been adapting its workforce availability to the various TAs, making successive experiences on employees' local changes. For the average case considered to $\mathrm{TA}_{5}$, his proposal corresponds to a $112 \mathrm{~h}$ workforce, representing $696.64 €$, using the same kind of 3-8 h shifts and verifying the same quality criteria described and used to build Formulation 2. This represents a gap of $7.14 \%$, when compared with the best solution proposed in this paper.

This comparison shows not only the great interest of the optimization formulation presented in this paper but also the confirmation that the solution proposed by the manager to this $\mathrm{TA}_{5}$ was not far from the best he could afford to, although the $104 \mathrm{~h}$ solution shows that he could have gone further.

In a different perspective, it is also interesting to analyse the case where only $8 \mathrm{~h}$ working shifts are in operation. By adapting Formulation 2 to that particular situation, we obtain an optimal solution of $945.44 €$ on daily salaries, using $152 \mathrm{~h}$ of total workforce. This means that by using the $3-8 \mathrm{~h}$ shifts solutions, we can reduce significantly the human resources needs in this particular TA.

(ii) Comparison between small, average and high amount cases: In this point, we analyse the average case: $Q=46925$, and also small and high daily amounts of mail-objects entered at $\mathrm{TA}_{5}$. The small and high amount cases are the first and the third quartiles, namely $Q=36709$ and 55006 , 
Table 4

Workforce solutions per block determined to the $Q_{1}, \bar{Q}$ and $Q_{3}$ mail arrival scenarios

\begin{tabular}{|c|c|c|c|c|c|c|c|c|c|c|c|c|}
\hline Blocks & $\begin{array}{l}17: 00- \\
18: 00\end{array}$ & $\begin{array}{l}18: 00- \\
19: 00\end{array}$ & $\begin{array}{l}19: 00- \\
20: 00\end{array}$ & $\begin{array}{l}20: 00- \\
21: 00\end{array}$ & $\begin{array}{l}21: 00- \\
22: 00\end{array}$ & $\begin{array}{l}22: 00- \\
23: 00\end{array}$ & $\begin{array}{l}23: 00- \\
0.00\end{array}$ & $\begin{array}{l}0: 00- \\
1: 00\end{array}$ & $\begin{array}{l}1: 00- \\
2: 00\end{array}$ & $\begin{array}{l}2: 00- \\
3: 00\end{array}$ & $\begin{array}{l}3: 00- \\
4: 00\end{array}$ & Total \\
\hline$Q_{1}$ & 0 & 2 & 11 & 17 & 16 & 7 & 0 & 0 & 10 & 10 & 10 & 83 \\
\hline $\bar{Q}$ & 0 & 3 & 16 & 22 & 21 & 6 & 0 & 0 & 12 & 12 & 12 & 104 \\
\hline$Q_{3}$ & 1 & 1 & 15 & 25 & 24 & 11 & 0 & 0 & 15 & 15 & 15 & 122 \\
\hline
\end{tabular}

Table 5

MIP solver information related with the three mail arrival scenarios: $Q_{1}, \bar{Q}$ and $Q_{3}$

\begin{tabular}{llll}
\hline Amounts entered & Lower bound $(€)$ & Upper bound & Duality gap $(\%)$ \\
\hline$Q_{1}$ & 501.80 & $516.30 €=83 \mathrm{~h}$ & 2.89 \\
$\bar{Q}$ & 638.69 & $646.88 €=104 \mathrm{~h}$ & 1.27 \\
$Q_{3}$ & 739.52 & $758.88 €=122 \mathrm{~h}$ & 2.55 \\
\hline
\end{tabular}

respectively. We designate the three mentioned cases by $\bar{Q}=46925, Q_{1}=36709$ and $Q_{3}=55006$, respectively.

Table 4 presents the number of employees per block in the best feasible solution obtained by the MIP solver, after $12 \mathrm{~h}$ of running time. The standard quality criterion has been kept at a $99 \%$ level. Like the $\bar{Q}$ case, the other two quantities analysed $\left(Q_{1}\right.$ and $\left.Q_{3}\right)$ require only 3 and $4 \mathrm{~h}$ shifts.

In Table 5, the lower and upper bound limits obtained after $12 \mathrm{~h}$ of the branch-and-bound execution, and the associated duality gaps, are reported.

These results show that a workforce ranging between 83 and $122 \mathrm{~h}$ can answer $50 \%$ of the 251 observations of daily mail income amounts. This means that the $122 \mathrm{~h}$ workforce solution is sufficient to treat $75 \%$ of the mentioned 251 observations, when the quality criteria is established at a $99 \%$ standard. This result may introduce a new discussion, that is, how far could we go, answering to the 251 daily observations, with the same $122 \mathrm{~h}$ of workforce, if the quality criteria standard is decreased? To answer this question, we have analysed unitary decreases on the quality criteria value, from $99 \%$ to $88 \%$, where the objective is to determine the maximum amount of mail-objects allowed to enter to $\mathrm{TA}_{5}$ and treated, at the quality standard established, by a $122 \mathrm{~h}$ workforce. This analysis is similar to a previous one, proposed in point (i). It also involves a changed version of Formulation 2, which includes the same non-negative variable $\beta$, defining the proportional increase in the 55006 amount of mail-objects. The modified formulation involves the maximization of $\beta$, subject to Formulation 2 constraints, together with equation $\sum_{k=1}^{K} \delta_{k} v_{k}=122$ ( $\delta_{k}$ is the time duration, in hours, of shift $k$ ) and substituting all the parameters $q_{i j}$ by $q_{i j} \beta$, in constraints (11a)-(13b). Table 6 presents the best feasible solutions found to each of the approaches, from $99 \%$ to $88 \%$ cases, after $12 \mathrm{~h}$ of the branch-and-bound execution. The accomplishment columns represent the percentage of accomplishment achieved over the 251 daily observations. To each daily amount observation $Q$, the mentioned accomplishment is considered at the quality criteria level $(\xi)$ established, so considering that $Q \xi$ mail-objects are treated. 
Table 6

MIP solver information related with the twelve quality criteria standards: $99-88 \%$, imposing the use of $122 \mathrm{~h}$ of workforce. Mail-objects (m-o) are rounded to the closest integer value

\begin{tabular}{|c|c|c|c|c|c|}
\hline $\begin{array}{l}\text { Workforce }= \\
122 \mathrm{~h}, \text { quality } \\
\text { criteria }(\xi)(\%)\end{array}$ & Lower bound & $\begin{array}{l}\text { Accom- } \\
\text { plishment } \\
(\%)\end{array}$ & Upper bound & $\begin{array}{l}\text { Accom- } \\
\text { plishment } \\
(\%)\end{array}$ & $\begin{array}{l}\text { Duality } \\
\text { gap (\%) }\end{array}$ \\
\hline 99 & $\beta \geqslant 1.008=55466 \mathrm{~m}-\mathrm{o}$ & 76 & $\beta \leqslant 1.018=55996 \mathrm{~m}-\mathrm{o}$ & 77 & 0.98 \\
\hline 98 & $\beta \geqslant 1.014=55776 \mathrm{~m}-\mathrm{o}$ & 77 & $\beta \leqslant 1.059=58251 \mathrm{~m}-\mathrm{o}$ & 83 & 4.25 \\
\hline 97 & $\beta \geqslant 1.041=57249 \mathrm{~m}-\mathrm{o}$ & 79 & $\beta \leqslant 1.083=59595 \mathrm{~m}-\mathrm{o}$ & 86 & 3.88 \\
\hline 96 & $\beta \geqslant 1.061=58361 \mathrm{~m}-\mathrm{o}$ & 83 & $\beta \leqslant 1.106=60837 \mathrm{~m}-\mathrm{o}$ & 89 & 4.07 \\
\hline 95 & $\beta \geqslant 1.092=60064 \mathrm{~m}-\mathrm{o}$ & 86 & $\beta \leqslant 1.126=61941 \mathrm{~m}-\mathrm{o}$ & 90 & 3.02 \\
\hline 94 & $\beta \geqslant 1.101=60562 \mathrm{~m}-\mathrm{o}$ & 88 & $\beta \leqslant 1.142=62817 \mathrm{~m}-\mathrm{o}$ & 91 & 3.59 \\
\hline 93 & $\beta \geqslant 1.118=61497 \mathrm{~m}-\mathrm{o}$ & 90 & $\beta \leqslant 1.158=63697 \mathrm{~m}-\mathrm{o}$ & 92 & 3.45 \\
\hline 92 & $\beta \geqslant 1.153=63447 \mathrm{~m}-\mathrm{o}$ & 91 & $\beta \leqslant 1.180=64907 \mathrm{~m}-\mathrm{o}$ & 93 & 2.29 \\
\hline 91 & $\beta \geqslant 1.176=64687 \mathrm{~m}-\mathrm{o}$ & 93 & $\beta \leqslant 1.194=65677 \mathrm{~m}-\mathrm{o}$ & 94 & 1.51 \\
\hline 90 & $\beta \geqslant 1.194=65695 \mathrm{~m}-\mathrm{o}$ & 94 & $\beta \leqslant 1.210=66557 \mathrm{~m}-\mathrm{o}$ & 94 & 1.32 \\
\hline 89 & $\beta \geqslant 1.212=66667 \mathrm{~m}-\mathrm{o}$ & 94 & $\beta \leqslant 1.224=67327 \mathrm{~m}-\mathrm{o}$ & 95 & 0.98 \\
\hline 88 & $\beta \geqslant 1.224=67327 \mathrm{~m}-\mathrm{o}$ & 95 & $\beta \leqslant 1.242=68317 \mathrm{~m}-\mathrm{o}$ & 95 & 1.45 \\
\hline
\end{tabular}

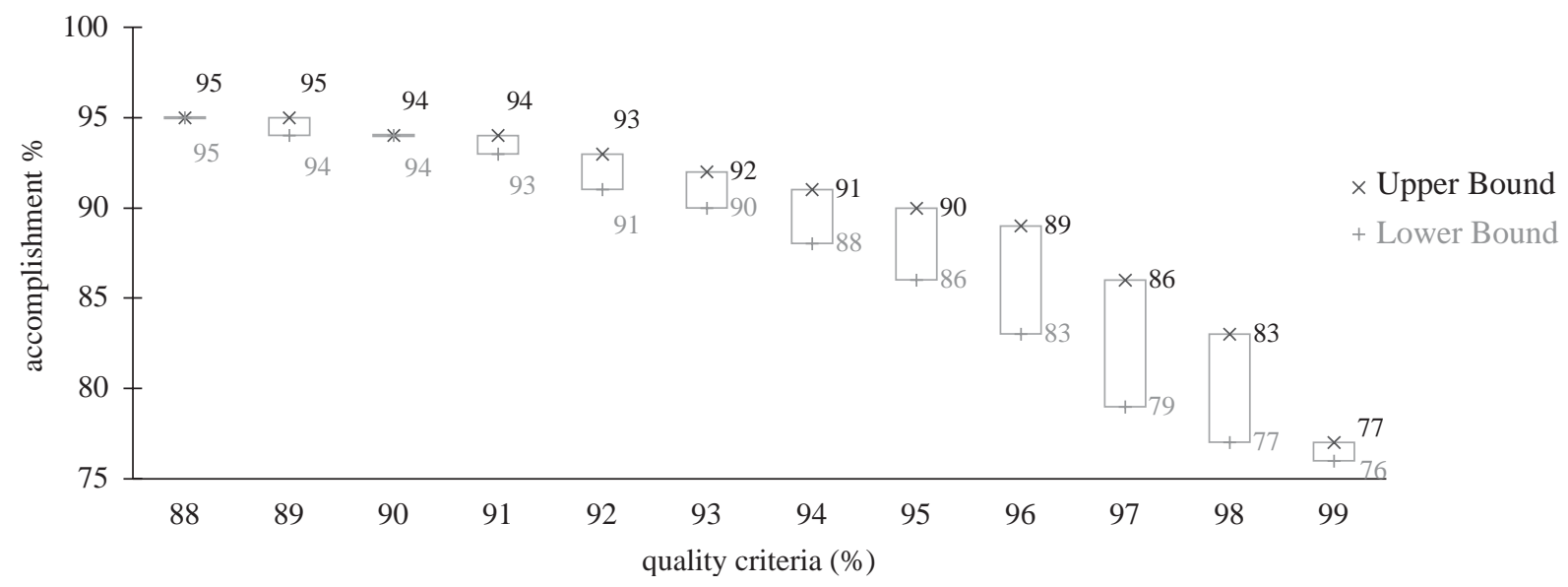

Fig. 8. Lower and upper bound values of the accomplishment to each quality criteria level, ranging from $99 \%$ to $88 \%$, with a fixed workforce of $122 \mathrm{~h}$.

Fig. 8 presents the lower and upper bound values of the accomplishment achieved according to unitary variations in the quality criteria level, considering the results in Table 6.

As expected, when the quality criteria level is allowed to be decreased, the $122 \mathrm{~h}$ of workforce are sufficient to answer to a higher number of days, in the 251 universe. Namely, for a quality criteria level of $99 \%$, the 122 workforce hours are sufficient to answer to at least $76 \%$ of the days, while if that quality standard is allowed to be relaxed to $93 \%$, then the same workforce can answer up to $90 \%$ of the days. If only $5 \%$ of the days are allowed to fail with the quality criteria established, than this standard must be relaxed to $88 \%$, still using the same workforce. 
Table 7

MIP solver information related with the two scenarios: $Q^{-}$and $Q^{+}$

\begin{tabular}{llll}
\hline Daily mail amounts & Lower bound & Upper bound & Duality gap (\%) \\
\hline$Q^{-}=25947$ & $376.50 € \rightarrow 61 \mathrm{~h}$ & $379.42 €=61 \mathrm{~h}$ & 0.77 \\
$Q^{+}=69039$ & $914.82 € \rightarrow 147 \mathrm{~h}$ & $933.00 €=150 \mathrm{~h}$ & 1.95 \\
\hline
\end{tabular}

We recall that the 122 workforce hours solution has been suggested to answer the $Q_{3}$ amount of mail case, being sufficient to guarantees a $99 \%$ quality criteria on $75 \%$ of the days. The previous result shows that we can go further, establishing that the same manpower level can answer at least $76 \%$ of the days, still keeping the same quality criteria standard.

(iii) Weeks with small and high average amounts of mail arrived: The previous analyses are related with daily income mail amounts, based on the 251 daily observations of the year 2001. As mentioned in Section 2.2, in practice, $\mathrm{TA}_{5}$ employees' assignment plan is established for each week, according to the amounts of mail expected to be received during that week. In this point, and also in the next one, year 2001 data observations are going to be analysed again, but this time looking for its weekly behaviour.

In those data values, the median observation in the weeks with smaller and higher average mail income amounts are $Q^{-}=25947$ and $Q^{+}=69039$ mail-objects, respectively. These two peak weeks may be seen as low and hard workforce weeks in human resources needs. The low week case can be observed in August during the typical vacations period, when most Public Institutions are closed. The high week peak occurs in December and may be related with Christmas time and with the proximity of the end of the year. Remember that $\mathrm{TA}_{5}$ only treats registered mail.

In Table 7, the lower and upper bound limits obtained after $12 \mathrm{~h}$ of the branch-and-bound execution and the associated duality gaps, are reported. A lower limit on manpower usage has been calculated through the expression $\lceil($ lower bound)/6.22 $\rceil$ (using the hourly cost of work, equal to $6.22 €)$.

The results define extreme values in human resources needs, according to small and high weekly income mail amounts. Those limits indicate that only 61 workforce hours are needed in low peak periods (e.g., in August), while in high peak periods the system may require $150 \mathrm{~h}$ of workforce. These solutions refer to the $99 \%$ quality criteria standard. Resorting to an analysis described in point (ii), the $Q^{+}=69039$ daily mail arrived case belongs to the $5 \%$ worse observations which, for a workforce of $122 \mathrm{~h}$, does not even allow to reach an $88 \%$ level on the quality criteria.

Considering the 61 and 150 workforce hours proposed to both cases $Q^{-}$and $Q^{+}$, respectively, it is interesting to analyse the way the system reacts when facing the highest amount observations occurring inside each one of those weeks. We recall that the values initially considered correspond to the median among each week observations. In the first case (in $Q^{-}$week) the highest daily income amount is equal to 28366 mail-objects, while in the second case (in $Q^{+}$week) that value is equal to 77950 mail-objects. Considering appropriate changes in Formulation 2 and solving the adapted model, we can state that the 61 workforce hours can treat up to 27581 mail-objects, corresponding to a $98 \%$ quality criteria; while in the second case, the 150 workforce hours allow to treat 74888 mail-objects, corresponding to a $96 \%$ quality criteria. 
Table 8

MIP solver information related with the weeks with shortest and highest differences between the maximum and the median observations, respectively 1003 and 18363 mail-objects. (In both cases the model has been solved for the median observation)

\begin{tabular}{llllllll}
\hline & \multicolumn{2}{l}{ Mail-objects } & & \multicolumn{2}{l}{ Considering the median observation for mail income amount } \\
\cline { 2 - 3 } \cline { 6 - 7 } & Median & Maximum & Difference & & Lower bound & Upper bound \\
\hline Shortest difference & 58818 & 59821 & 1003 & & & $782.05 € \rightarrow 126 \mathrm{~h}$ & $802.38 €=129 \mathrm{~h}$ \\
Highest difference & 46421 & 64784 & 18363 & & $632.57 € \rightarrow 102 \mathrm{~h}$ & $646.88 €=104 \mathrm{~h}$ \\
\hline
\end{tabular}

(iv) Weeks with high and short error plans, in workforce assignment: Still centred in the weekly behaviour of year 2001 mail income observations, we analyse the errors that may be committed when trying to plan human resources needs for a week period. With this purpose and using the mentioned data observations, we analyse the weeks with highest and shortest differences between the maximum and the median observations. Four outliers are excluded from the discussion.

This analysis starts by determining the workforce required to answer the median amount case, to both weeks under consideration. Table 8, presents the lower and upper bound limits obtained after $12 \mathrm{~h}$ of the branch-and-bound execution. Again, the lower limit on manpower usage has been calculated through the expression $\lceil$ (lower bound)/6.22 (considering the hourly cost of work, equal to $6.22 €)$.

These results show that in both weeks, if the human resources intended to assigned to this TA are based on the median mail amount observation, then there should be proposed 129 workforce hours for the first case (shortest difference) and $104 \mathrm{~h}$ of workforce to the second one (highest difference). Both workforce resources should be kept during the whole week. The problem comes when we have to face the maximum amounts in that week with the proposed human requirements, and a question comes immediately: what is the quality criteria achieved if we use the proposed workforce ( $129 \mathrm{~h}$ to the first case and $104 \mathrm{~h}$ to the second one) to treat the maximum amount expected in the correspondent week? To answer this question, we considered a modified version of Formulation 2 , similar to another one used before, involving the maximization of the nonnegative variable $\phi$, representing the standard quality criteria, also appearing in constraints (13a) and (13b). The two cases under consideration (shortest and highest difference) require different formulations. For the first case (shortest difference), the model includes the constraint $\sum_{k=1}^{K} \delta_{k} v_{k}=129$, while for the second one (highest difference) the model considers $\sum_{k=1}^{K} \delta_{k} v_{k}=104$, where $\delta_{k}$ is the time duration, in hours, of shift $k$. Both formulations use different daily mail income amounts, namely $Q=59821$ and $Q=64784$, respectively, for the shortest and the highest difference cases.

Table 9 presents results related with both modified formulations, after $12 \mathrm{~h}$ of the branch-and-bound execution.

The results show that in the shortest difference case, the $99 \%$ quality criteria is still guaranteed, while in the highest difference case we may only achieve a quality criteria level of $81 \%$. This means that the Company may face a shortage in the delivery quality standard if high differences in daily mail amounts received are neglected, when the plan is established for an expected median scenario in manpower resources proposed to each week. 
Table 9

MIP solver information related with the two modified formulations, proposed to maximize the quality criteria achieved when facing maximum mail amount quantities with a workforce established according to the median observation mail amount, to both shortest and highest difference scenarios

\begin{tabular}{lllll}
\hline & Maximum & Workforce $(\mathrm{h})$ & Lower bound & Upper bound \\
\hline Shortest difference & $59821 \mathrm{~m}-\mathrm{o}$ & 129 & $\phi \geqslant 0.99=59256 \mathrm{~m}-\mathrm{o}$ & $\phi \leqslant 0.995=59500 \mathrm{~m}-\mathrm{o}$ \\
Highest difference & $64784 \mathrm{~m}-\mathrm{o}$ & 104 & $\phi \geqslant 0.81=52442 \mathrm{~m}-\mathrm{o}$ & $\phi \leqslant 0.815=52773 \mathrm{~m}-\mathrm{o}$ \\
\hline
\end{tabular}

It can be showed that the 64784 mail-objects can be treated by a workforce solution of $141 \mathrm{~h}$ (lower limit of $139 \mathrm{~h}$ ), with a guaranteed quality criteria of $99 \%$.

\section{Conclusions}

This paper describes an application to a batching decision process, in which the daily workforce cost of a mail processing center TA is minimized. A formulation to this problem has been proposed. A branch-and-bound algorithm has processed the resulting optimization problem and has been able to produce solutions, answering to many proposed scenarios. Those solutions have been considered important for decision making support to the Company Staff Department. Based in the year 2001 behaviour, workforce requirements were discussed for different daily mail amounts entered in $\mathrm{TA}_{5}$, and considering different levels for the quality criteria on daily total mail treated.

Other changes on the parameters could have been considered, namely variations on the type and duration of working shifts, periods of failure in some TUs and other scenarios that have not been discussed in this work.

The next step of this collaboration with CTT should involve the study of the other TAs, by adapting Formulation 1 to those specific treatment areas. The last step should incorporate in a single model all the TAs staff planning structure, characterizing a global environment and considering the employees interchange among TAs. This model is more challenging, especially from the optimization point of view, eventually requiring the use of decomposition techniques to obtain good solutions in a reasonable amount of time.

\section{Acknowledgements}

The authors are quite grateful to the referees for their comments and suggestions that have contributed to improve the quality of the paper. The research of the first and the second authors is partially supported by the projects FCT-POCTI/35059/MAT/2000 and POCTI/ISFL/152, respectively. 


\section{Appendix A}

$P 1$ matrix, where $p 1_{r i}$ is the mail-flow transfer rate from $\mathrm{TU}_{r}$ to $T U_{i}(r, i=1, \ldots, M)$ in the 17:00-23:00 period

$$
P 1=\left[\begin{array}{ccccccccc}
0 & 0.09 & 0.16 & 0.74 & 0 & 0.007 & 0 & 0 & 0 \\
0 & 0 & 0 & 0 & 0 & 0 & 0.46 & 0.54 & 0 \\
0 & 0 & 0 & 0 & 0 & 0 & 0.36 & 0.64 & 0 \\
0 & 0 & 0 & 0 & 0.47 & 0 & 0.25 & 0.28 & 0 \\
0 & 0 & 0 & 0 & 0 & 0 & 0.53 & 0.47 & 0 \\
0 & 0 & 0 & 0 & 0 & 0 & 0 & 1 & 0 \\
0 & 0 & 0 & 0 & 0 & 0 & 0 & 0 & 1 \\
0 & 0 & 0 & 0 & 0 & 0 & 0 & 0 & 1 \\
0 & 0 & 0 & 0 & 0 & 0 & 0 & 0 & 0
\end{array}\right] .
$$

$P 2$ matrix, where $P 2_{r i}$ is the mail-flow transfer rate from $\mathrm{TU}_{r}$ to $\mathrm{TU}_{i}(r, i=1, \ldots, M)$ in the 23:00-04:00 period

$$
P 2=\left[\begin{array}{ccccccccc}
0 & 0 & 0 & 0 & 0 & 0 & 0 & 0 & 0 \\
0 & 0 & 0 & 0 & 0 & 0 & 0 & 1 & 0 \\
0 & 0 & 0 & 0 & 0 & 0 & 0 & 1 & 0 \\
0 & 0 & 0 & 0 & 0.47 & 0 & 0 & 0.53 & 0 \\
0 & 0 & 0 & 0 & 0 & 0 & 0 & 1 & 0 \\
0 & 0 & 0 & 0 & 0 & 0 & 0 & 0 & 1 \\
0 & 0 & 0 & 0 & 0 & 0 & 0 & 0 & 1 \\
0 & 0 & 0 & 0 & 0 & 0 & 0 & 0 & 1 \\
0 & 0 & 0 & 0 & 0 & 0 & 0 & 0 & 0
\end{array}\right] .
$$

Parameters $q_{i j}(i=1, \ldots, M-1, j=1, \ldots, N)$ representing the number of mail-objects arrived directly to each TU per period are given below:

\begin{tabular}{lrlcccccc}
\hline \multicolumn{1}{l}{ Period } & TU1 & TU2 & TU3 & TU4 & TU5 & TU6 & TU7 & TU8 \\
\hline $1 \rightarrow 17: 00-17: 15$ & 2345 & 0 & 0 & 0 & 0 & 0 & 0 & 0 \\
$2 \rightarrow 17: 15-17: 30$ & 2345 & 0 & 0 & 0 & 0 & 0 & 0 & 0 \\
$3 \rightarrow 17: 30-17: 45$ & 0 & 0 & 0 & 0 & 0 & 0 & 0 & 0 \\
$4 \rightarrow 17: 45-18: 00$ & 0 & 0 & 0 & 0 & 0 & 0 & 0 & 0 \\
$5 \rightarrow 18: 00-18: 15$ & 0 & 0 & 0 & 0 & 0 & 0 & 0 & 0 \\
$6 \rightarrow 18: 15-18: 30$ & 0 & 0 & 0 & 0 & 0 & 0 & 0 & 0 \\
$7 \rightarrow 18: 30-18: 45$ & 0 & 0 & 0 & 0 & 0 & 0 & 0 & 0 \\
$8 \rightarrow 18: 45-19: 00$ & 3518 & 0 & 0 & 0 & 0 & 0 & 0 & 0 \\
$9 \rightarrow 19: 00-19: 15$ & 3518 & 0 & 0 & 0 & 0 & 0 & 0 & 0
\end{tabular}




\begin{tabular}{|c|c|c|c|c|c|c|c|c|}
\hline Period & TU1 & TU2 & TU3 & TU4 & TU5 & TU6 & TU7 & TU8 \\
\hline $10 \rightarrow 19: 15-19: 30$ & 3518 & 0 & 0 & 0 & 0 & 0 & 0 & 0 \\
\hline $11 \rightarrow 19: 30-19: 45$ & 3518 & 0 & 0 & 0 & 0 & 0 & 0 & 0 \\
\hline $12 \rightarrow 19: 45-20: 00$ & 0 & 0 & 0 & 0 & 0 & 0 & 0 & 0 \\
\hline $13 \rightarrow 20: 00-20: 15$ & 3881 & 0 & 0 & 0 & 0 & 0 & 0 & 0 \\
\hline $14 \rightarrow 20: 15-20: 30$ & 3881 & 0 & 0 & 0 & 0 & 0 & 0 & 0 \\
\hline $15 \rightarrow 20: 30-20: 45$ & 4951 & 0 & 0 & 0 & 0 & 0 & 0 & 0 \\
\hline $16 \rightarrow 20: 45-21: 00$ & 4952 & 0 & 0 & 0 & 0 & 0 & 0 & 0 \\
\hline $17 \rightarrow 21: 00-21: 15$ & 1055 & 0 & 0 & 0 & 0 & 0 & 0 & 0 \\
\hline $18 \rightarrow 21: 15-21: 30$ & 1055 & 0 & 0 & 0 & 0 & 0 & 0 & 0 \\
\hline $19 \rightarrow 21: 30-21: 45$ & 0 & 0 & 0 & 0 & 0 & 0 & 0 & 0 \\
\hline$\cdots$ & $\ldots$ & $\cdots$ & $\cdots$ & $\ldots$ & $\cdots$ & $\cdots$ & $\cdots$ & $\cdots$ \\
\hline $37 \rightarrow 02: 00-02: 15$ & 0 & 0 & 0 & 0 & 0 & 0 & 0 & 0 \\
\hline $38 \rightarrow 02: 15-02: 30$ & 0 & 0 & 0 & 0 & 0 & 0 & 0 & 0 \\
\hline $39 \rightarrow 02: 30-02: 45$ & 0 & 0 & 450 & 2078 & 0 & 8 & 260 & 0 \\
\hline $40 \rightarrow 02: 45-03: 00$ & 0 & 0 & 450 & 2078 & 0 & 8 & 260 & 0 \\
\hline $41 \rightarrow 03: 00-03: 15$ & 0 & 0 & 450 & 2078 & 0 & 8 & 260 & 0 \\
\hline $42 \rightarrow 03: 15-03: 30$ & 0 & 0 & 0 & 0 & 0 & 0 & 0 & 0 \\
\hline $43 \rightarrow 03: 30-03: 45$ & 0 & 0 & 0 & 0 & 0 & 0 & 0 & 0 \\
\hline $44 \rightarrow 03: 45-04: 00$ & 0 & 0 & 0 & 0 & 0 & 0 & 0 & 0 \\
\hline
\end{tabular}

\section{Appendix B}

Table 10 presents the number of employees determined per block and assigned to each TU: variables $w_{i h}$ values.

Table 10

Number of employees determined per block and assigned to each TU: variables $w_{i h}$ values

\begin{tabular}{|c|c|c|c|c|c|c|c|c|}
\hline \multirow[t]{2}{*}{ One hour blocks } & \multicolumn{8}{|c|}{ Employees in each TU $\left(w_{i h}\right)$} \\
\hline & TU1 & TU2 & TU3 & TU4 & TU5 & TU6 & TU7, 8 & Total \\
\hline 17:00-18:00 & 0 & 0 & 0 & 0 & 0 & 0 & 0 & 0 \\
\hline 18:00-19:00 & 1 & 0 & 1 & 1 & 0 & 0 & 0 & 3 \\
\hline $19: 00-20: 00$ & 2 & 1 & 1 & 7 & 2 & 1 & 2 & 16 \\
\hline 20:00-21:00 & 3 & 2 & 0 & 9 & 5 & 1 & 2 & 22 \\
\hline 21:00-22:00 & 1 & 1 & 3 & 6 & 3 & 0 & 7 & 21 \\
\hline $22: 00-23: 00$ & 0 & 0 & 1 & 0 & 1 & 1 & 3 & 6 \\
\hline 23:00-00:00 & 0 & 0 & 0 & 0 & 0 & 0 & 0 & 0 \\
\hline 00:00-01:00 & 0 & 0 & 0 & 0 & 0 & 0 & 0 & 0 \\
\hline 01:00-02:00 & 0 & 0 & 0 & 0 & 0 & 0 & 12 & 12 \\
\hline 02:00-03:00 & 0 & 0 & 1 & 6 & 2 & 0 & 3 & 12 \\
\hline 03:00-04:00 & 0 & 0 & 1 & 3 & 2 & 1 & 5 & 12 \\
\hline
\end{tabular}




\section{References}

[1] Kuik R, Salomon M. Multi-level lot-sizing problem: evaluation of a simulated-annealing heuristic. European Journal of Operational Research 1990;45:25-37.

[2] Maes J, McClain JO, van Wassenhove LN. Multilevel capacitated lotsizing complexity and LP-based heuristics. European Journal of Operational Research 1991;53:131-48.

[3] Tempelmeier H, Helber S. A heuristic for dynamic multi-item multi-level capacitated lotsizing for general product structures. European Journal of Operational Research 1994;75:296-311.

[4] Stadler H. Mixed integer programming model formulations for dynamic multi-item multi-level capacitated lotsizing. European Journal of Operational Research 1996;94:561-81.

[5] Tempelmeier H, Derstroff M. A Lagrangean-based heuristic for dynamic multi-level multi-item constrained lotsizing with setup times. Management Science 1996;42:738-57.

[6] Kimms A, Drexl A. Proportional lot sizing and scheduling: some extensions. Networks 1998;32:85-101.

[7] Kuik R, Salomon M, van Wassenhove LN. Batching decisions: structure and models. European Journal of Operational Research 1994;75:243-63.

[8] Drexl A, Kimms A. Lot sizing and scheduling-survey and extensions. European Journal of Operational Research 1997;99:221-35.

[9] Wolsey L. Solving multi-item lot-sizing problems with an MIP solver using classification and reformulation. Management Science 2002;48:1587-602.

[10] Belvaux G, Wolsey L. Modelling practical lot-sizing problems as mixed-integer programs. Management Science 2001;47(7):993-1007.

[11] Bahl HC, Ritzman LP, Gupta JND. Determining lot sizes and resource requirements: a review. Operations Research 1987;35:329-45.

[12] Pochet Y, Wolsey LA. Solving multi-item lot sizing problems using strong cutting planes. Management Science 1991;37:53-67.

[13] Shapiro JF. Mathematical programming models and methods for production planning and scheduling. In: Graves SC, Rinnooy Kan AHG, Zipkin PH (editors). Handbooks in operations research and management science. Logistics of production and inventory. vol. 4. Amsterdam: North-Holland; 1993 [chapter 8].

[14] Belvaux G, Wolsey L. Lot-sizing problems: modelling issues and a specialized branch-and-cut system BC-PROD. Core Discussion Paper 9849, 1998.

[15] Pochet Y. Mathematical programming models for deterministic production planning problems. In: Jünger M, Naddef D, editors. Computational combinatorial optimization. Lecture notes in computer science, vol. 2241. Berlin, Heidelberg: Springer; 2001. p. 57-111.

[16] Bechtold SE, Jacobs LW. The equivalence of general set-covering and implicit integer programming formulations for shift scheduling. Naval Research Logistics 1996;43:233-49.

[17] Aykin T. A comparative evaluation of modelling approaches to the labor shift scheduling problem. European Journal of Operational Research 2000;125:381-97.

[18] Caprara A, Monaci M, Toth P. Models and algorithms for a staff scheduling problem. Mathematical Programming Series B 2003;98:445-76.

[19] Mehrotra A, Murphy KE, Trick MA. Optimal shift scheduling: a branch-and-price approach. Naval Research Logistics 2000;47:185-200.

[20] Tien JM, Kamiyama A. On manpower scheduling algorithms. SIAM Review 1982;24(3):275-87.

[21] Ernst AT, Jiang H, Krishnamoorthy M, Sier D. Staff scheduling and rostering: a review of applications, methods and models. European Journal of Operational Research 2004;153:3-27.

[22] Bodin L, Golden B, Assad A, Ball M. Routing and scheduling of vehicles and crews-the state of the art. Computers and Operations Research 1983;10(2):63-211.

[23] Caprara A, Fischetti M, Toth P, Vigo D, Guida PL. Algorithms for railway crew management. Mathematical Programming 1997;79:125-41.

[24] Vance PH, Barnhart C, Johnson EL, Nemhauser GL. Airline crew scheduling: a new formulation and decomposition algorithm. Operations Research 1997;45(2):188-200.

[25] Gamache M, Soumis F, Marquis G, Desrosiers J. A column generation approach for large-scale aircrew rostering problems. Operations Research 1999;47(2):247-63. 
[26] Dawid H, König J, Strauss C. An enhanced rostering model for airline crews. Computers and Operations Research 2001;28:671-88.

[27] Fischetti M, Lodi A, Martello S, Toth P. A polyhedral approach to simplified crew scheduling and vehicle scheduling problems. Management Science 2001;47(6):833-50.

[28] Dijkstra MC, Kroon LG, Salomon M, van Nunen JAEE, van Wassenhove LN. Planning the size and organization of KLM's aircraft maintenance personnel. Interfaces 1994;24(6):47-58.

[29] Brusco MJ, Jacobs LW, Bongiorno RJ, Lyons DV, Tang B. Improving personnel scheduling at airline stations. Operations Research 1995;43(5):741-51.

[30] Mason AJ, Ryan D, Panton DM. Integrated simulation, heuristic and optimisation approaches to staff scheduling. Operations Research 1998;46(2):161-75.

[31] Segal M. The operator-scheduling problem: a network-flow approach. Operations Research 1974;22:808-23.

[32] Lin CKY, Lai KF, Hung SL. Development of a workforce management system for a customer hotline service. Computers and Operations Research 2000;27:987-1004.

[33] Balakrishnan N, Wong RT. A network model for the rotating workforce scheduling problem. Networks 1990;20: $25-42$.

[34] Jaumard B, Semet F, Vovor T. A generalized linear programming model for nurse scheduling. European Journal of Operational Research 1998;107:1-18.

[35] Bellanti F, Carello G, Della Croce F, Tadei R. A greedy-based neighborhood search approach to a nurse rostering problem. European Journal of Operational Research 2004;153:28-40.

[36] Malhotra MK, Ritzman LP, Benton WC, Keong Leong G. A model for scheduling postal distribution employees. European Journal of Operational Research 1992;58:374-85.

[37] Jarrah AIZ, Bard JF, deSilva AH. Solving large-scale tour scheduling problems. Management Science 1994;40(9):1124-44.

[38] Bard JF, Binici C, deSilva AH. Staff scheduling at the United States Postal Service. Computers and Operations Research 2003;30:745-71.

[39] Ilog Inc. ILOG CPLEX User's Manual, Version 7.0. Ilog Inc., 2000.

[40] SPSS 11.0 Applications guide. Chicago IL: SPSS Inc.; 2002. 